\title{
Methodological Considerations on the Use of Different Spectral Decomposition Algorithms to Study Hippocampal Rhythms
}

\author{
Y. Zhou, ${ }^{1}{ }^{\circledR}$ A. Sheremet, ${ }^{1,2}$ Y. Qin, ${ }^{1}$ J. P. Kennedy, ${ }^{2}$ N. M. DiCola, ${ }^{2}$ ' S. N. Burke, ${ }^{2}$ and ${ }^{\circledR}$ A. P. Maurer ${ }^{1,2,3}$ \\ https://doi.org/10.1523/ENEURO.0142-19.2019 \\ ${ }^{1}$ Engineering School of Sustainable Infrastructure and Environment, University of Florida, Gainesville, FL 32611, \\ ${ }^{2}$ McKnight Brain Institute, Department of Neuroscience, University of Florida, Gainesville, FL 32610, and ${ }^{3}$ Department \\ of Biomedical Engineering, University of Florida, Gainesville, FL 32611
}

\begin{abstract}
Local field potential (LFP) oscillations are primarily shaped by the superposition of postsynaptic currents. Hippocampal LFP oscillations in the 25- to $50-\mathrm{Hz}$ range ("slow $\gamma$ ") are proposed to support memory retrieval independent of other frequencies. However, $\theta$ harmonics extend up to $48 \mathrm{~Hz}$, necessitating a study to determine whether these oscillations are fundamentally the same. We compared the spectral analysis methods of wavelet, ensemble empirical-mode decomposition (EEMD), and Fourier transform. EEMD, as previously applied, failed to account for the $\theta$ harmonics. Depending on analytical parameters selected, wavelet may convolve over high-order $\theta$ harmonics due to the variable time-frequency atoms, creating the appearance of a broad $25-$ to $50-\mathrm{Hz}$ rhythm. As an illustration of this issue, wavelet and EEMD depicted slow $\gamma$ in a synthetic dataset that only contained $\theta$ and its harmonics. Oscillatory transience cannot explain the difference in approaches as Fourier decomposition identifies ripples triggered to epochs of high-power, $120-$ to $250-\mathrm{Hz}$ events. When Fourier is applied to high power, 25- to $50-\mathrm{Hz}$ events, only $\theta$ harmonics are resolved. This analysis challenges the identification of the slow $\gamma$ rhythm as a unique fundamental hippocampal oscillation. While there may be instances in which slow $\gamma$ is present in the rat hippocampus, the analysis presented here shows that unless care is exerted in the application of EEMD and wavelet techniques, the results may be misleading, in this case misrepresenting $\theta$ harmonics. Moreover, it is necessary to reconsider the characteristics that define a fundamental hippocampal oscillation as well as theories based on multiple independent $\gamma$ bands.
\end{abstract}

Key words: EEMD; low $\gamma$; nonlinearity; velocity

\section{Significance Statement}

Fourier, Wavelet and ensemble empirical-mode decomposition (EEMD) converge on conflicting representations of the same time series. Fourier reveals $\theta$ harmonics whereas wavelet and EEMD have identified a "slow $\gamma$ " oscillation. In a comparison of spectral decomposition methods, we find that wavelet and EEMD give the erroneous impression of a slow $\gamma$ band. Fourier decomposition does not display any spectral deviation that is indicative of slow $\gamma$. These data emphasize the importance of multiple analytical approaches with well-understood parameters when decomposing LFP in relation to behavior. On a fundamental level, our analysis points to an imprecise definition of a fundamental rhythm, requiring a reconsideration of both characteristics that define a hippocampal oscillation as well as theories based on multiple $\gamma$ bands. 


\section{Introduction}

Oscillations in the local field potential (LFP) were initially hypothesized to be organized in an energy cascade framework (Bak et al., 1987; Buzsáki and Draguhn, 2004; Buzsáki, 2006). In this framework, higher amplitude, lower frequency rhythms provide the energy into lower amplitude-higher frequency rhythms, suggesting that every frequency is interdependent. Activity across all frequencies reflects a single unified process. This theory had a significant amount of traction, explaining how the crossscale energy cascade is responsible for the redistribution of power across frequencies and the increase in $\theta-\gamma$ coupling with velocity (Ahmed and Mehta, 2012; Sheremet et al., 2019a,b).

The energy cascade hypothesis, however, was all but abandoned following the report of independent "slow $\gamma$ " $(25-50 \mathrm{~Hz})$ and "high $\gamma$ " (65-140 Hz) bands (Colgin et al., 2009). The slow $\gamma$ rhythm identified in the CA1 pyramidal layer correlated with activity in the CA3 region, whereas the higher frequency $\gamma$ rhythm showed coherence with the medial entorhinal cortex. This led to the two $\gamma$ hypothesis by which each rhythm is statistically independent and supports dissociable psychological processes (Colgin, 2015b).

The identification of two independent $\gamma$ rhythms was predicated on a cross-frequency coherence analysis using a Morlet wavelet estimation of power and frequency, showing coherence of slow and fast $\gamma$ with the $\theta$ rhythm (Colgin et al., 2009, their Fig. 1C; n.b., $50-\mathrm{Hz}$ alternating current noise overlaps with the $\theta \times$ slow $\gamma$ interaction in this figure). Other decomposition methods, however, only found interactions between $\theta$, a single $\gamma(30-80 \mathrm{~Hz})$ and a $120-$ to $160-\mathrm{Hz}$ oscillation (Zhang et al., 2016), or $\theta, \theta$ harmonics and a single $\gamma$ (Sheremet et al., 2019a, their Fig. 6), failing to observe interactions between $\theta$ and the slow $\gamma$ band. Other absences in the detection of slow $\gamma$ include cross-frequency power correlations or bicoherence analyses (Buzsáki et al., 2003; Masimore et al., 2004; Johnson and Redish, 2007; Sheremet et al., 2016, 2019a). These studies, however, have been eclipsed by reports on slow $\gamma$ that have reconfirmed the phenomenon through wavelet decomposition (Belluscio et al., 2012; Lasztóczi

Received April 13, 2019; accepted July 12, 2019; First published July 19, 2019. The authors declare no competing financial interests.

Author contributions: Y.Z., A.S., S.N.B., and A.P.M. designed research; Y.Z., A.S., Y.Q., J.P.K., N.M.D., and A.P.M. analyzed data; Y.Z., A.S., S.N.B., and A.P.M. wrote the paper; J.P.K. performed research.

This work was supported by the McKnight Brain Research Foundation, by National Institutes of Health (NIH) National Institute on Aging Grant AG055544 and NIH National Institute of Mental Health Grant MH109548, and by a Diversity Supplement to NIH Grant R01MH109548 (to J.P.K.).

Acknowledgements: We thank S. D. Lovett for technical support. We appreciate the time, feedback and dialog provided by our reviewers.

Correspondence should be addressed to A. P. Maurer at drewmaurer@ufl.edu.

https://doi.org/10.1523/ENEURO.0142-19.2019

Copyright (C) 2019 Zhou et al.

This is an open-access article distributed under the terms of the Creative Commons Attribution 4.0 International license, which permits unrestricted use, distribution and reproduction in any medium provided that the original work is properly attributed. and Klausberger, 2014, 2016; Tamura et al., 2017; Zhong et al., 2017; Dvorak et al., 2018).

To fully appreciate the equivocal foundation of slow $\gamma$, it pays to examine the lack of consistency in its described properties across studies. Although harmonics of $\theta$ were described relatively early (Harper, 1971) and noted to extend into the 32- to $40-\mathrm{Hz}$ band (Leung et al., 2005), they were not isolated in the initial description of slow $\gamma$ (Colgin et al., 2009). Furthermore, there is a notable incongruence between the contemporarily defined ranges of slow $\gamma$ bands across publications (Fig. 1). For instance, the slow $\gamma$ range defined by Schomburg et al. (2014), $30-80 \mathrm{~Hz}$, broadens the initial range identified by Colgin et al. (2009) and precisely mirrors the filter parameters of Csicsvari et al. (2003), which describes a single $\gamma$ band. Reverting the filter parameters to ranges used in earlier reports obfuscates the phenomenon being discussed and points to an incongruency in how we define a fundamental hippocampal rhythm. As "fundamental rhythms" are often defined by a substantial "peak above noise" observation (Pesaran et al., 2018; e.g., why spectral whitening is often implemented), it is necessary to understand the initial rationale behind subdividing $\gamma$ an how this relates to the harmonics of $\theta$, which extend as high as $48 \mathrm{~Hz}$ (Sheremet et al., 2016).

The current work revisits prior decomposition techniques employed to identify slow $\gamma$ frequencies. Our investigation demonstrates that different analysis methods result in contradictory results. Ensemble empirical-mode decomposition (EEMD), as implemented by Lopes-DosSantos et al. (2018), fails to account for the higher order harmonics of $\theta$. The analytical parameters chosen in the wavelet analysis of Colgin et al. (2009) produce a corrupt representation of the high-order $\theta$ harmonics leading to the appearance of a broad $25-$ to $50-\mathrm{Hz}$ deflection in the power spectrum. In contrast, $\theta$ harmonics are readily identified by short-timescale Fourier decomposition. Critically, spectral decomposition methods as contemporarily implemented yield distinct representations of the same data. To resolve these incongruencies, these three decomposition methods were run on a synthetic benchmark dataset. Notably, both Fourier and wavelet with larger temporal support yielded results closer to the known parameters than EEMD or standard wavelet parameters. The current observations suggest that it is necessary to consider if decomposition methods are appropriately applied and to implement multiple analytical approaches with well-understood parameters when decomposing time-series signals in relation to behavior.

\section{Materials and Methods}

\section{Subjects and behavioral training}

All behavioral and surgical procedures were performed in accordance with the National Institutes of Health guidelines for rodents and protocols approved by the University of Florida Institutional Animal Care and Use Committee. LFP data were obtained from four- to 10-month-old Fisher 344xBrown Norway F1 hybrid rats from the National Institute on Aging colony (Charles River). Datasets used to compare spectral decompositions methods were ob- 


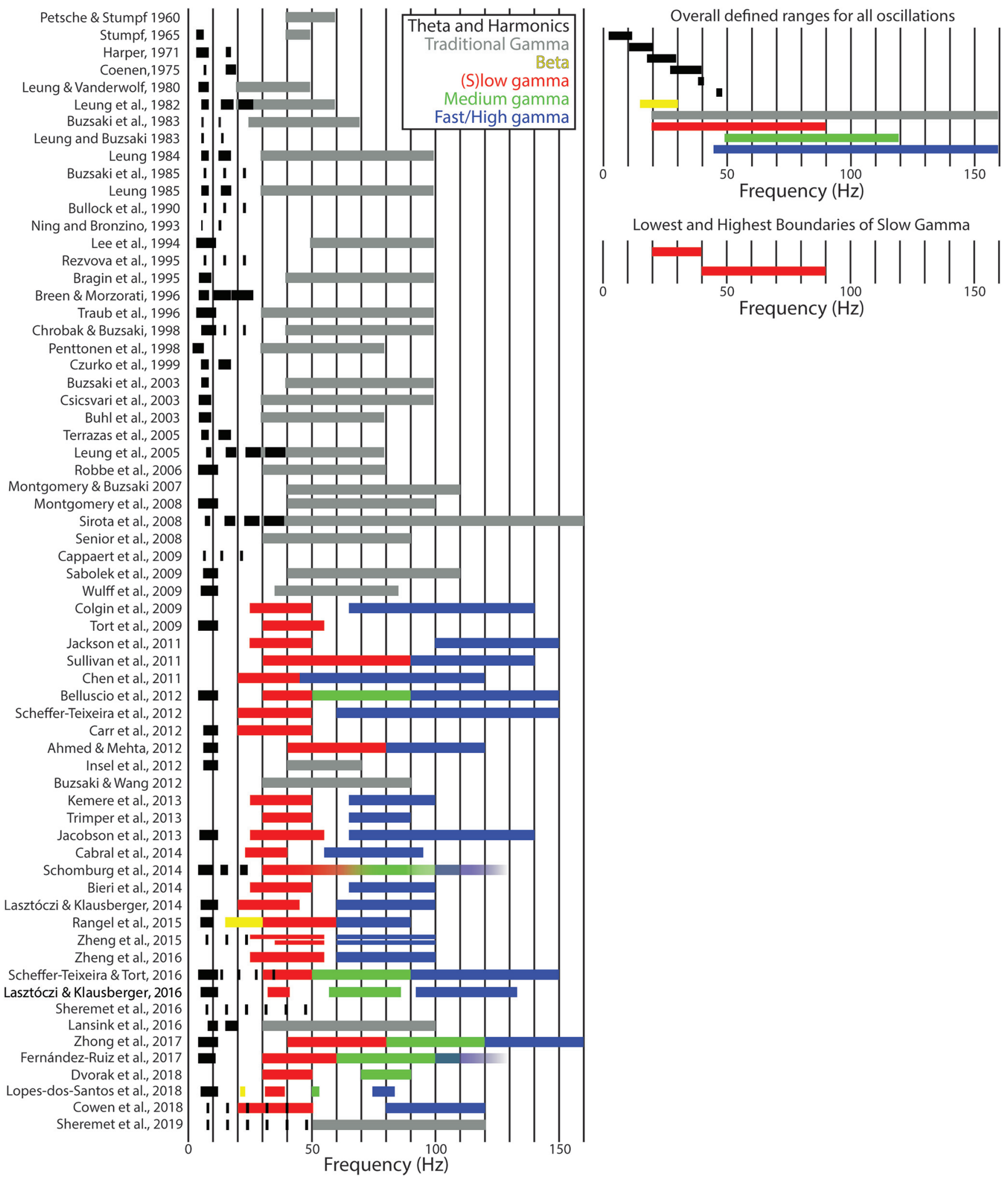

Figure 1. Left, Historical definition of medial temporal lobe oscillatory bands. For each manuscript, a bar spans the range defined for a specific oscillation. In the instance that a range is not defined, but an oscillation is noted (Lopes-Dos-Santos et al., 2018; Sheremet et al., 2019a), a marker covering a short frequency range was used. Furthermore, some ranges were inferred (Sirota et al., 2008 describe a high $32-$ to $40-\mathrm{Hz}$ harmonic, indicative that the $16-$ and $24-\mathrm{Hz}$ subdivisions also exist). Note that, before the subdivision of $\gamma$ into multiple bands, multiple harmonics of $\theta$ were reported. For example, Leung et al. (1982) verified the presence of harmonics through bicoherence analysis. Following the observation of multiple $\gamma$ bands, few manuscripts account for the harmonics of $\theta$. The notable exceptions are Schomburg et al. (2014), cautioning that pyramidal neuron spike modulation in the $20-$ to $30-\mathrm{Hz}$ band may be 
continued

related to the third harmonic of $\theta$ and not slow $\gamma$, and Scheffer-Teixeira and Tort (2016) cautioned that $\theta$ wave asymmetry may erroneously contribute to cross-frequency coupling. Cowen et al. (2018) observed up to the fifth harmonic $(40+\mathrm{Hz})$ in both old and young rats which overlapped with their slow $\gamma$ range, but did not clarify the difference between harmonics and slow $\gamma$. Zheng et al. (2015) also increased the lower bound of their slow $\gamma$ definition in the medial entorhinal cortex in an attempt to avoid the lower order $\theta$ harmonics. The recent implementation of bicoherence analysis, however, reveals that the harmonics of $\theta$ can extend as high as 48 $\mathrm{Hz}$ (Sheremet et al., 2016, 2019a). Note that before the discovery of slow $\gamma$, only $\theta$ harmonic were reported in the $25-$ to $50-\mathrm{Hz}$ range. Following the slow $\gamma$ discovery, reports of harmonics became rare. Right top, An examination of the most extensive defined ranges of all oscillations. Note that $\theta$ harmonics spill into the lowest range of traditional and (s)low $\gamma$. Therefore, it is evident that harmonics potentially contribute to the $\gamma$ band up to $50 \mathrm{~Hz}$. No attempt has been made to decipher if the medium $\gamma$ (Belluscio et al., 2012) was equivalent to fast/high $\gamma$ across manuscripts. Right bottom, Perhaps more concerning is that when examining the lowest defined ranges (defined by identifying the lowest high pass and the lowest low pass ranges), the (s)low $\gamma$ band does not overlap across studies, demonstrating inconsistency. Critically, a meaningful definition of what is a fundamental rhythm is missing. Black: $\theta$ and $\theta$ harmonics; gray: "fast oscillation" and non-subdivided $\gamma$; yellow: $\beta$; red: (s)low $\gamma$; green: medium $\gamma$; blue: fast/high $\gamma$ (Petsche and Stumpf, 1960; Stumpf, 1965; Harper, 1971; Coenen, 1975; Leung and Vanderwolf, 1980; Leung, 1982, 1984, 1985; Buzsáki et al., 1983, 1985, 2003; Leung and Buzsáki, 1983; Bullock et al., 1990; Ning and Bronzino, 1993; Lee et al., 1994; Bragin et al., 1995; Rezvova et al., 1995; Breen and Morzorati, 1996; Traub et al., 1996; Chrobak and Buzsáki, 1998; Penttonen et al., 1998; Czurkó et al., 1999; Buhl et al., 2003; Csicsvari et al., 2003; Leung et al., 2005; Terrazas et al., 2005; Robbe et al., 2006; Montgomery and Buzsáki, 2007; Montgomery et al., 2008; Senior et al., 2008; Sirota et al., 2008; Cappaert et al., 2009; Colgin et al., 2009; Sabolek et al., 2009; Tort et al., 2009; Wulff et al., 2009; Chen et al., 2011; Jackson et al., 2011; Sullivan et al., 2011; Ahmed and Mehta, 2012; Belluscio et al., 2012; Buzsáki and Wang, 2012; Carr et al., 2012; Insel et al., 2012; Scheffer-Teixeira et al., 2012; Jacobson et al., 2013; Kemere et al., 2013; Bieri et al., 2014; Cabral et al., 2014; Lasztóczi and Klausberger, 2014, 2016; Schomburg et al., 2014; Trimper et al., 2014; Rangel et al., 2015; Zheng et al., 2015, 2016; Lansink et al., 2016; Scheffer-Teixeira and Tort, 2016; Sheremet et al., 2016, 2019a; Fernández-Ruiz et al., 2017; Zhong et al., 2017; Cowen et al., 2018; Dvorak et al., 2018; Lopes-Dos-Santos et al., 2018).

tained from three rats (mixed sex cohort: 2 male, 1 female). On arrival, rats acclimated to the colony room for one week. The rats were housed and maintained on a $12 / 12 \mathrm{~h} \mathrm{light/dark}$ cycle. All training sessions and electrophysiological recordings took place during the dark phase of the rats' light/dark cycle. Training consisted of shaping the rats to traverse a circular track for food reward $(45 \mathrm{mg}$, unflavored dustless precision pellets; product \#F0021, BioServ). During this time, their body weight was slowly reduced to $85 \%$ of their free-feeding baseline. Once the rat reliably performed more than one lap per minute, they were implanted with a custom single shank silicon probe from NeuroNexus or Cambridge NeuroTech. The NeuroNexus probe was designed such that thirty-two recording sites, each with a recording area of $177 \mu \mathrm{m}$, were spaced $60 \mu \mathrm{m}$ apart allowing incremental recording across the hippocampal lamina. The Cambridge NeuroTech probe consisted of sixty-four recording sites, each with an area of $165 \mu \mathrm{m}$ and spaced $50 \mu \mathrm{m}$ apart, allowing for $3.15 \mathrm{~mm}$ of vertical coverage. In preparation for surgery, the probes were cleaned in a $4 \%$ dilution of Contrad detergent (Decon Contrad 70 Liquid Detergent, Fisher Scientific) and then rinsed in distilled water.

\section{Surgical procedures}

Rats were initially sedated in an induction chamber containing 3-5\% isoflurane. Once anesthetized, the rat was moved to a nose cone, the head was shaved, and the rat was transferred to the stereotax. During surgical implantation, the rats were maintained under anesthesia with isoflurane administered at doses ranging from 0.5 to $2.5 \%$. The probe implant coordinates targeted the dorsal hippocampus (AP: $-3.2 \mathrm{~mm}$, ML: 1.5 relative to bregma, DV: $-3.7 \mathrm{~mm}$ from brain surface). Once the location of the implant was identified, a $3 \times 3-\mathrm{mm}$ contour was drilled out around these coordinates, but not completed. This was followed by the placement of seven anchor screws in the bone as well as a reference over the cerebellum and ground screw placed over the cortex. Once the screws were secured, a thin layer of adhesive cement (C\&BMetabond, Parkell) followed by dental acrylic [Grip Cement Industrial Grade, 675571 (powder) 675572 (solvent); Dentsply Caulk] were applied taking care to not obscure the craniotomy location. Finally, the craniotomy location was completed, irrigating and managing bleeding as necessary once the bone fragment was removed. Next, a portion of the dura was removed, taking care to avoid damaging the vessels and the surface of the neocortex. Small bleeding was managed with saline irrigation and gel foam (sterile absorbable gelatin sponges manufactured by Pharmacia \& Upjohn Co). Once the probes were in place, the craniotomy was covered with SILASTIC (KwikSil, World Precision Instruments) and then secured to the anchor screws with dental acrylic. Four copper mesh flaps were placed around the probe providing protection as well as acting as a potential Faraday cage. The wires from the reference and ground screws were soldered to the appropriate pins of the electrode interface board. Adjacent regions of the copper-mesh flaps were soldered together to ensure their electrical continuity, and the ground wire soldered to the copper mesh taking care to isolate the reference from contact with the ground. Once the probe was secured, the rat received $10 \mathrm{cc}$ of sterile saline as well as Metacam $(1.0 \mathrm{mg} / \mathrm{kg})$ subcutaneously (the non-steroidal anti-inflammatory is also known as meloxicam; Boehringer Ingelheim Vetmedica, Inc.). The rat was placed in a cage and monitored until fully recovered. Over the next $7 \mathrm{~d}$, the rat was observed to ensure recovery and no behavioral anomalies. Metacam was administered the day following surgery as well. Antibiotics (sulfamethoxazole/trimethoprim oral suspension at 200 $\mathrm{mg} / 40 \mathrm{mg}$ per $5 \mathrm{ml}$; Aurobindo Pharma USA, Inc.) were administered in the rat mash for an additional $5 \mathrm{~d}$. 


\section{Neurophysiology}

Following recovery from surgery, rats were retrained to run unidirectionally on a circle (outer diameter: $115 \mathrm{~cm}$, inner diameter: $88 \mathrm{~cm}$ ), or figure- 8 track $(112 \mathrm{~cm}$ wide $\times$ $91 \mathrm{~cm}$ length) for food reward at a single location. The local-field potential was recorded on a Tucker-Davis Neurophysiology System at $24 \mathrm{kHz}$ (PZ2 and RZ2, TuckerDavis Technologies). The animal's position was recorded at $30 \mathrm{frames} / \mathrm{s}$ (Tucker-Davis Technologies). Spatial resolution was $<0.5 \mathrm{~cm} /$ pixel. Running speed was calculated as the derivative of the smoothed position. The LFP data were analyzed in MATLAB (MathWorks) using customwritten code as well as code imported from the HOSAtoolbox. Raw LFP records sampled at $24 \mathrm{kHz}$ (TuckerDavis System) were down-sampled to $2 \mathrm{kHz}$ and divided into fragments of 2048 time samples $(\sim 1 \mathrm{~s})$. To eliminate the effects of anatomic variations in electrode depth between rats, the position of the hippocampal layers with respect to the recording channels was determined by estimating the distribution of current-source density to identify the CA1 pyramidal cell layer (Rappelsberger et al., 1981; Mitzdorf, 1985; Buzsáki et al., 1986; Bragin et al., 1995). Unless otherwise noted, all decomposition analyses were conducted on LFP traces from the CA1 pyramidal cell layer.

\section{Additional LFP datasets}

To investigate cross-frequency interactions and as to not be redundant with previously published data from our lab, LFP recordings from two additional rats from the Buzsáki laboratory were included (https://buzsakilab. nyumc.org/datasets/FernandezRuiz_Oliva/AB1/day11/; https://buzsakilab.nyumc.org/datasets/FernandezRuiz Oliva/AYA1/AYA1_140808/; sex unknown). The electrode configuration of these probes consisted of eight shanks with 32 sites per shank. Electrode position was determined using current source density analyses (Rappelsberger et al., 1981; Mitzdorf, 1985; Buzsáki et al., 1986; Bragin et al., 1995) triggered to detected ripple events. The distribution of the current sources and sinks of the raw LFP triggered to ripples matched the regional distribution of activity to input layers (Ylinen et al., 1995; Sullivan et al., 2011; Sheremet et al., 2019a). Following current source density analysis, a single channel was selected in the CA1 pyramidal layer of each rat.

\section{Fourier and wavelet transforms}

\section{Transforms}

The Fourier and wavelet transform can be introduced formally as decomposition on a set of "elementary" functions. In the case of the Fourier transform, the functions form a basis. In the case of the wavelet transform, the most common elementary function sets form frame, a class with weaker properties than a basis. Procedures to extract orthogonal subsets from wavelet frames are available (e.g., the diadic construction; Mallat, 1999). Without going into the details, let $S$ be some class of real functions and let $\psi_{f}$, with $(f \in R$ ), be a basis in $S$; then any $g \in S$ can be written uniquely as

$$
G(f)=\int g(t) \psi_{f}(t) d t, g(t)=\int G(f) \psi_{f}(t) d f .
$$

The "coefficients" $G(f)$ of the decomposition (also referred to as the transform of $g$ ) are obtained by taking the inner product of the function $g$ with the basis elements [i.e., projecting $g(t)$ onto the basis], The pair of equations (Eqs. 2, 3) are best known as direct and inverse transforms, sometimes also called analysis and synthesis.

The Fourier transform pair is obtained letting $\psi_{f}(t)=$ e2 $2^{\text {mift }}$

$$
G(f)=\int_{-\infty}^{\infty} g(t) e^{-2 \pi i f t} d t, g(t)=\int_{-\infty}^{\infty} G(f) e^{2 \pi i f t} d f .
$$

The wavelet transform and its inverse are given by

$$
G(s, \tau)=\int_{-\infty}^{\infty} g(t) \psi_{s \tau}(t) d t, g(t)=\int_{-\infty}^{\infty} \int_{-\infty} G(s, \tau) \psi_{s \tau}(t) d s d \tau,
$$

where the functions $\psi_{s, \tau}(t)=\alpha \varphi(t-\tau / s)$ are "copies" of the "mother wavelet" $\varphi(t)$, shifted in time by $\tau$ and scaled by $s$, with $\alpha$ a normalization constant. The full set of wavelets $\left\{\psi_{s, \tau}(t)\right\}_{s, \tau \in R}$ is in general complete but not independent (i.e., larger than a basis). The wavelet representation was designed for two main applications: compression and resolving short-time transient coherent structures such as solitons and chirps. For these purposes, the lack of orthogonality of the decomposing modes is not an inconvenience, because the structure of the decomposition in the dual space is not important. Compression algorithms only need a small number of modes and the synthesis rule for reconstructing the signal, while the identification of transients focuses on optimizing the time-scale localization (see the discussion of the time-scale atoms below). The lack of orthogonality of the wavelet elementary function set creates problems in applications where one seeks to assign meaning to the actual components of the decomposition. Here, meaning means repetition (an implied stochastic process model) and requires the ability to attribute variance unequivocally to each of the decomposition modes. For such an application, the lack of orthogonality of the wavelet decomposition becomes a major inconvenience, because one cannot attribute unique variance to any single wavelet mode.

\section{Discrete transforms}

Unless the set of functions are carefully defined, Equations 2, 3 are only formal, and in most cases, they do not have any elementary mathematical meaning; when they do, their usefulness is limited. For example, if $g(t)=1$, the Riemann integral in the equation does not exist; however, a rather restrictive elementary theory can be built for $T$-periodic functions.

Evolving the generic Fourier and wavelet equations into useful analysis tools has complexities that required the development of full theories (e.g., the theory of distributions, or generalized functions; Schwartz, 1950; Lighthill, 1958). For example, in Fourier equations $\psi_{f}(t)=e^{2 \pi i f t}$ are 
orthogonal in the sense that $\int^{\infty} e^{2 \pi i f t} d t=\delta(f)$, where $\delta$ is the Dirac delta function (Vladimirov, 2002; Strichartz, 2003). A complete discussion of these is far beyond the scope of this study, and is also unnecessary, because time series measured in practical applications are always of finite length $T$, sampled at time intervals $\Delta t(T=n \Delta t)$, that is., are finite sequences of real numbers $g(t)=\left\{g_{1}, g_{2}, \cdots, g_{N}\right\}$, with $g_{j}=g\left(t_{j}\right)$. Such sequences naturally form $N$-dimensional spaces, in which integral Transforms, such as the Fourier or wavelet equations, are represented by finite-dimensional linear operators, that is, $N \times N$ matrices. These discretized versions of the Fourier and wavelet representations are called the discrete transforms (Briggs, 1995; Mallat, 1999; Strang, 2006).

The discrete Fourier transform pair is (in MATLAB convention)

$$
G_{m}=\frac{1}{N} \sum_{n=0}^{N-1} g_{n} \psi_{m n}, g_{n}=\sum_{m=0}^{N-1} G_{m} \psi_{m n}^{*}, \psi_{m n}=e^{-2 \pi i \frac{m n}{N}}
$$

where $\psi_{m n}=\psi_{m}\left(t_{n}\right)=e^{-2 \pi i f_{m} t_{n}}$ are the basis vectors. This pair of equationsare sometimes called the analysis and synthesis of the signal $g(t)$.

Here, $f_{m}=m \Delta f$ and $t_{n}=n \Delta t$ represent the discretized frequency and time grids, with $\Delta f=1 / T$, and $\Delta t=$ $T / N$. Basis functions are orthogonal in the sense that, for any integerm, with $m \neq 0$ and $m \neq N, \sum_{n=0}^{N-1} e^{-2 \pi i m n} / N=0$.

A discrete version of wavelet transform is

$$
G_{m n}=\sum_{k=0}^{N-1} g_{k} \varphi_{m n k}, \operatorname{with} \varphi_{m n k}=s^{-\frac{m}{2}} \varphi\left[s^{-m}\left(k-n \Delta \tau s^{m}\right)\right]
$$

where $\Delta \tau$ is a time-shift increment. The wavelets $\varphi_{m n k}$ form orthogonal only for compact-support wavelet shapes $\varphi$ (e.g., Haar, and Daubechies wavelets; Daubechies, 1988, 1992; Mallat, 1999).

The Parseval relation ensures that the discrete Fourier equations conserve the variance of the time sequence and its transform (Briggs, 1995), i.e., $\sigma^{g}=\sigma^{G}$, where $\sigma^{g}=$ $\Sigma_{n}\left|g_{n}\right|^{2}$ is the variance of $g$. The discrete wavelet transform does conserve variance $\left(\sigma^{g} \neq \sigma^{G}\right)$ and in general, the variance ratio depends on $g$, which means that a universal correction factor does not exist.

\section{Windowed Fourier transform (WFT)}

To investigate the evolution of spectra over time, WFT is introduced where the original signal is multiplied by a window function which is nonzero for only a short period of time

$$
G^{w i n}(\tau, f)=\int_{-\infty}^{\infty} g(t) w(t-\tau) e^{-2 \pi i t} d t
$$

where $w$ is the window function (Priestley, 1981; Roads, 2004). The window function slides along the time axis, which gives rise to a time-frequency representation of the original time series. This time-frequency representation can be plotted as the spectrogram. Typically, window functions are smooth, "bell-shaped," time-localized curves, the Fourier transform of the window function is

$$
W(f)=\int_{-\infty}^{\infty} w(t) e^{-2 \pi i f t} d t
$$

In frequency space, the window function $w$ is usually composed of a bell-shaped main lobe and symmetric side lobes. The bandwidth of a window function is defined as

$$
B=\frac{\int_{-\infty}^{\infty}|W(f)|^{2} d f}{|W(f)|_{\max }^{2}}
$$

The spectrum estimation depends critically on the window bandwidth as it will smooth the spectrum and influence the frequency resolution (Fig. 2). The time-frequency resolution of transforms will be further discussed in the following section.

\section{EEMD}

A brief description of empirical mode decomposition (EMD) is given in Figure 3, where the original time series is decomposed into intrinsic mode functions and a nonoscillatory residual. The intrinsic mode functions generated by EMD have two properties: (1) the number of extrema and the number of zero-crossings will either be equal or differ at most by one; (2) the upper envelope and lower envelope are symmetric. These two properties guarantee the intrinsic mode functions have a wellbehaved Hilbert transform. However, the decomposing process doesn't ensure orthogonality of intrinsic mode functions, and except for those two properties, we have limited prior knowledge about the decompose results. The question, why the symmetric mode functions are favorable, and why a well-behaved Hilbert transform is desired should be asked before applying the EMD. However, EMD suffers from the mode mixing problem which renders the algorithm unstable (Gledhill, 2003). To solve this issue, EEMD is proposed. In the EEMD algorithm, white noise is added to the original signal before decomposition to alleviate mode mixing and is canceled out after the decomposition by averaging over ensemble (Wu and Huang, 2009). In this study, following the procedure of Lopes-Dos-Santos et al. (2018), the ratio between the variance of added white noise and the original LFP was 0.5 , and the ensemble number was 200. After applying the EEMD, the power spectrum for each intrinsic mode function was used to identify the peak frequency. The supra- $\theta$ signal is defined as the sum of all the intrinsic mode functions with peak frequency above $12 \mathrm{~Hz}$. The timescale power distribution of supra- $\theta$ signal was obtained by Morlet wavelet transform with a constant ratio for the wavelet family set as 7 (Colgin et al., 2009; the meaning of this parameter is introduced in the numerical implementation section).

Time-frequency atoms

For the Fourier transform, the duration $T$ of the time 

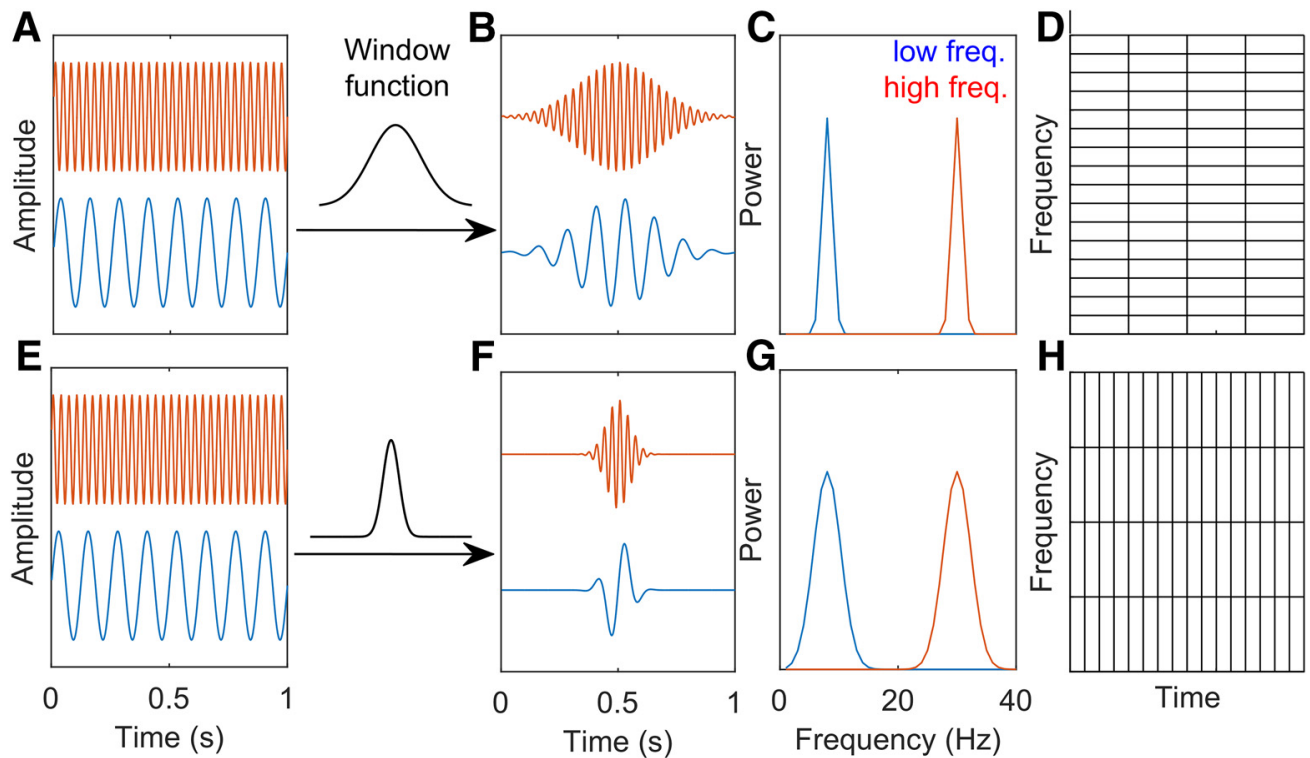

Time (s)

Time (s)

Figure 2. WFT atoms. $\boldsymbol{A}$, For WFT, a window function is applied to all the frequency components. $\boldsymbol{B}$, Adoption of a Hanning window with wide time support to fast and slow oscillations, which $(\boldsymbol{C})$ gives rise to fast and slow oscillations that decay slowly in the time domain. $\boldsymbol{D}$, In the frequency domain, the power (amplitude square) of these slowly decaying oscillations have a relatively narrow frequency band, and the bandwidth is the same for fast and slow oscillations. This results in a transform with coarse time resolution but fine frequency resolution. $\boldsymbol{E}$, On the contrary, applying a Hanning window with narrow time leads to $(\boldsymbol{F})$ fast decay oscillations in the time domain. $\mathbf{G}$, However, these fast decay oscillations have a wide frequency band, which $(\boldsymbol{H})$ results in a transform with fine time resolution but coarse frequency resolution.

sequence and the frequency resolution $\Delta f$ of the transformed sequence are related through the reciprocity relation $T \Delta f=1$, which implies that increasing the frequency resolution $\Delta f$ is equivalent to increasing the time duration $T$ of the analyzed signal. The $T \Delta f=1$ equation highlights an important limitation of the Fourier transform: if $g(t)$ is highly localized, its transform $G(t)$ has a wide frequency support. This means that very high sampling rates cover the wide frequency domain, and the interpretation of the high-frequency content can become difficult. Restricting the Fourier analysis equation to a specified duration $T$ is equivalent to multiplying the time series $g(t)$ by finite support rectangular window $w(t-\tau)=1$ if $|t-\tau| \leq T / 2$ and zero otherwise. In other words, the integral operator

$$
\int_{\tau-T / 2}^{\tau+T / 2} d t e^{-2 \pi i f t}
$$

may be written as

$$
\int_{-\infty}^{\infty} d t \& w(t-\tau) e^{-2 \pi i f t}=\int_{-\infty}^{\infty} d t \varphi_{f \tau}^{*}(t)
$$

which may be interpreted as a projection of $g$ onto functions $\varphi(t)=w(t) \psi(t)$. The function $\varphi$ could be described as a localized oscillation. If one constructs in the timefrequency plane a rectangle of sides $T$ and $\Delta f$ centered, say, at $t_{0}=T / 2$ and $f_{0}=T \Delta f / 2 \Delta t$, the $T \Delta f=1$ equation states that the area of this rectangle is constant, regardless of the value of $T$. This rectangle is sometimes called the Heisenberg box (Mallat, 1999). This is in fact an ex- ample of the application of the general Heisenberg uncertainty principle, which states that the area of a Heisenberg box cannot be made arbitrarily small. For an arbitrarilyshaped localized oscillation $\varphi(t)$ with Fourier transform $\varphi(f)$ and unit variance $\left(\int_{-\infty}^{\infty}|\varphi|^{2} d t=\int_{-\infty}^{\infty}|\phi|^{2} d t=1\right)$, defining the time and frequency ${ }^{-\infty}$ widths as

$$
\sigma_{t}=\int_{-\infty}^{\infty}\left(t-t_{0}\right)^{2}|\varphi|^{2} d t, \quad \sigma_{f}=\int_{-\infty}^{\infty}\left(f-f_{0}\right)^{2}|\phi|^{2} d t,(11)
$$

one can show (Gabor, 1946; Percival and Walden, 1993; Mallat, 1999) that $\sigma_{t} \sigma_{f} \geq 1 / 4 \pi$.

In other words, it is impossible to achieve simultaneous arbitrary resolutions both in time and frequency. While the time-frequency resolution (area of Heisenberg boxes) cannot be made arbitrarily small, it can be minimized. Gabor (1946) showed that the minimal area for a Heisenberg box is achieved by localized oscillation $\varphi(t)=$ $w(t) e^{2 \pi i f t}$ where $w$ is a Gaussian-shaped window. Following his work, Goupillaud et al. (1984) used this shape, later called the Morlet wavelet, to introduce the continuous wavelet transform (Grossmann and Morlet, 1984; Mallat, 1999). The limitations imposed by the Heisenberg uncertainty principle are illustrated in Figure 4. A Morlet wavelet results as a product of a sine function with a Gaussian window. The resulting function is the mother wavelet. The wavelet transform then uses scaled versions of the mother wavelet as elementary functions. It is important to note that the wavelets shown in Figure $4 D$, are not harmonic; therefore, they are not uniquely characterized by a single frequency value; instead, they are characterized by 

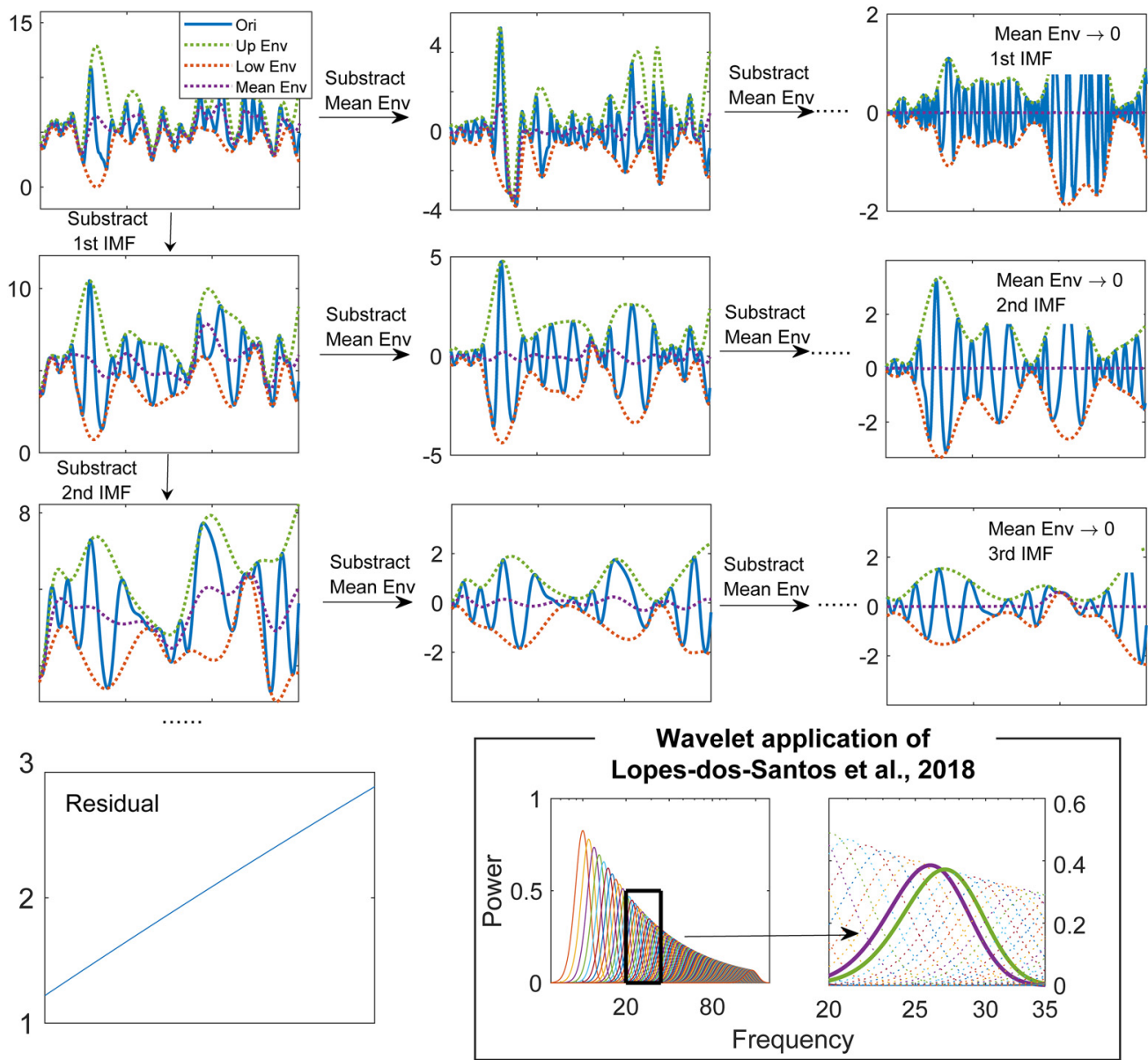

Figure 3. Description of empirical mode decomposition and the wavelet analysis as used by Lopes-Dos-Santos et al. (2018). For a given time series, the mean envelope can be obtained by averaging upper envelope and lower envelope. Subtracting the mean envelope from the original time series and repeating this process until the time series has an almost symmetric upper and lower envelopes. The obtained time series is defined as the first order intrinsic mode function (the 1st row in the figure). Subtracting the 1st intrinsic mode function from the time series and repeating the process, we can get high order intrinsic mode functions (the 2 nd and the 3rd rows). Following this process, the time series will be decomposed into intrinsic mode functions and a residual, which has overlapped upper and lower envelopes. Inset, Rather than use the wavelet steps of Colgin et al. (2009), Lopes-Dos-Santos et al. (2018) opted to use steps of $1 \mathrm{~Hz}$ between Morlet wavelets making for a highly redundant, overly convolved representation.

a frequency interval, say, corresponding to the width of the frequency spread of their power (as given by the Fourier transform). The frequency distribution of power is, as expected, narrower for the larger-scale wavelets, and wider for smaller-scale wavelets. Therefore, the wavelet Heisenberg boxes coverage of the frequency axis is not uniform, and consequently, using wavelets as a "frequency" representation results in a non-uniform frequency resolution, with resolution degrading at higher frequencies.

It is worth noting that, theoretically, wavelet transform can acquire arbitrary frequency resolution at a given frequency range by dilating the width of the mother wavelet. However, an appropriate choice of mother wavelet requires a good understanding of the process under investigation. In the analysis of $\theta$ harmonics, if we want to obtain the frequency resolution of $2 \mathrm{~Hz}$ when the central frequency is around $30 \mathrm{~Hz}$, the mother wavelet will have $\sim 20$ oscillations before decaying (compare Fig. $4 D$, which is the typical wavelet used in the confirmation of slow $\gamma$ analysis). Thus, it is worth noting that wavelet is capable of resolving harmonics, but not in the implementation of Colgin et al. (2009).

\section{Numerical implementation}

Fourier and wavelet analysis procedures were implemented in the MATLAB environment using available MATLAB toolboxes. The Fourier spectrum was estimated by dividing the LFP time series into 2048-point (1 s) segments sorted by rat speed and windowed using a Hamming window. The wavelet transform used the Morlet wavelet, with the central frequency of the Morlet wavelet defined as the nominal frequency. The Morlet wavelet family is characterized by the constant ratio between the central frequency of wavelet and SD of the applied Gaussian window, which is set to 7 in our analysis to match the methods of Colgin et al. (2009).

The wavelet scalogram is defined as the logarithm of the squared modulus $\left|G_{m n}\right|^{2}$ of the wavelet coefficients. The power spectral density (PSD) for the wavelet trans- 

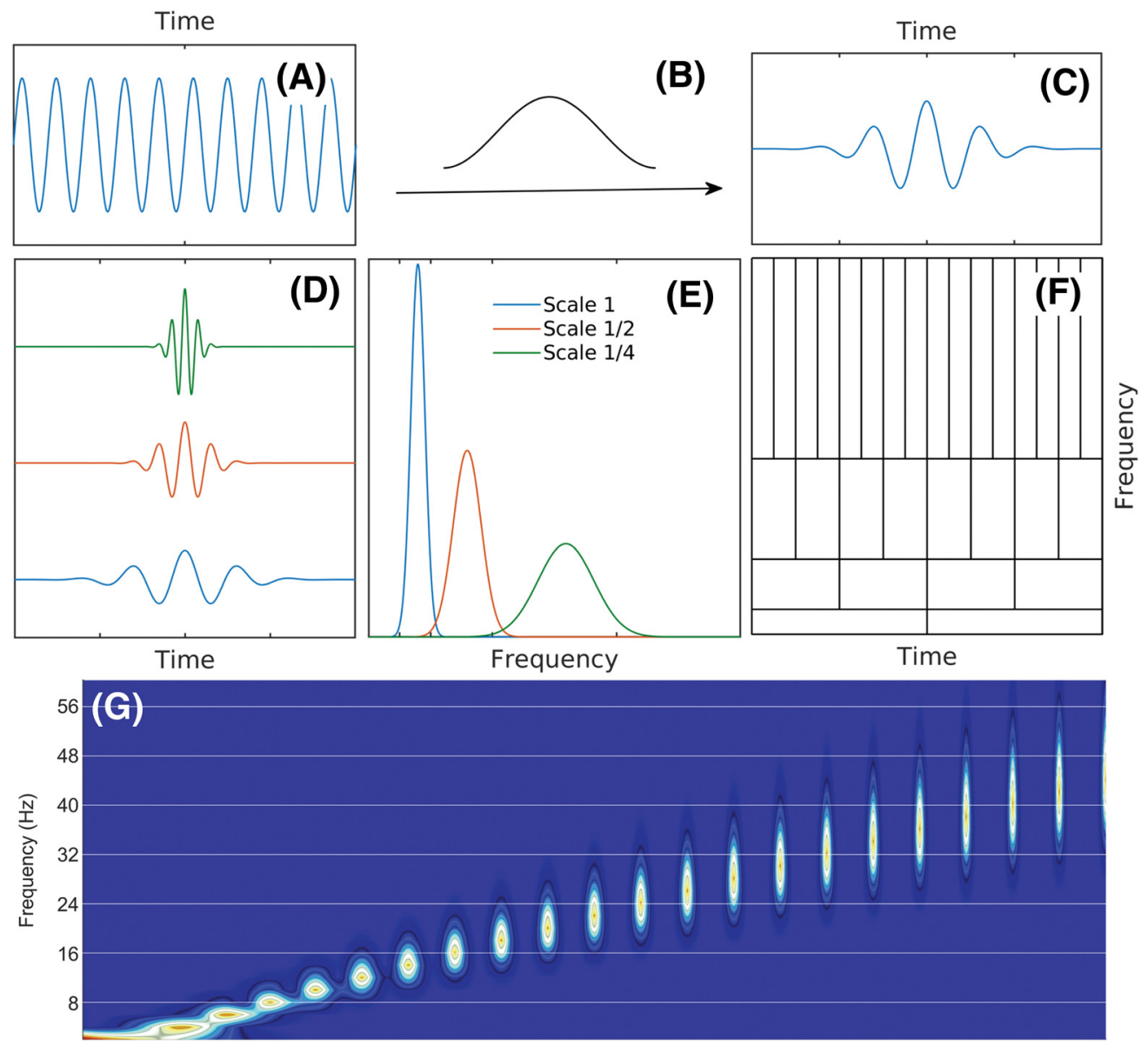

Figure 4. A schematic of the Heisenberg boxes of the wavelet transform. $\boldsymbol{A}-\boldsymbol{C}$, The mother wavelet is obtained by the multiplication of a sine function with a window. $\boldsymbol{D}$, Wavelets at three scales resulting by the scaling of the mother wavelet. $\boldsymbol{E}$, Frequency distribution of the power of wavelets. $\boldsymbol{F}$, Heisenberg boxes of the wavelet transform. $\boldsymbol{G}$, Frequency distributions of the first 22 wavelet scales used in Colgin et al. (2009). Note that the 8-Hz bin only overlaps with approximately three wavelets, $16 \mathrm{~Hz}$ overlap with nearly five wavelets, $24 \mathrm{~Hz}$ overlaps with approximately seven wavelets, and $32 \mathrm{~Hz}$ overlaps with more than 10 wavelets. It is this decimation that results in distributing the power of the $24-\mathrm{Hz}$ and higher harmonics of $\theta$ over multiple frequency bands. The implementation of Lopes-DosSantos (2018) accentuates this effect through their use of $1-\mathrm{Hz}$ steps between Morlet wavelets.

form was computed as the time marginal of the wavelet transform (Abry et al., 1993). The Fourier spectra were estimated using the Welch method (Welch, 1967).

\section{Methodological limitation}

At this point, it needs to be stressed that the wavelet and EEMD assignment of variance to a specific frequency (e.g., in the construction of a PSD) is ultimately arbitrary and potentially meaningless since the elementary functions used for the decompositions are not harmonics functions (sine/cosine). The data presented here are for illustrative and replication purposes. We believe this methodological treatment is flawed and should be treated with circumspection.

\section{Bicoherence analysis}

Higher-order spectra provide information regarding the degree of cross-frequency coupling. The bispectrum, the "lowest" of the high-order spectra, has had a long history in the field of wave dynamics (Hasselmann et al., 1963; Rosenblatt and Van Ness, 1965; Coppi et al., 1969). The bispectrum has been thoroughly reviewed in terms of both statistical and mathematical background (Harris, 1967) as well as its application to nonlinear wave interaction (Kim and Powers, 1979). Furthermore, the relationship between a time series and the third-order statistic is well understood (Haubrich and MacKenzie, 1965; Masuda and Kuo, 1981; Elgar, 1987). As noted previously (Sheremet et al., 2016; Kovach et al., 2018), bispectral analysis (the Fourier transform of the third-order cumulant) quantifies the degree of phase-envelope amplitude coupling between the frequencies of the LFP, while the bicoherence quantifies the degree of cross-frequency coupling independent from the amplitude (Barnett et al., 1971; Ning and Bronzino, 1989; Sigl and Chamoun, 1994; Bullock et al., 1997; Muthuswamy et al., 1999; Hagihira et al., 2001; Gloveli et al., 2005; Li et al., 2009; Wang et al., 2017; Shahbazi Avarvand et al., 2018). It is emphasized here as well as elsewhere (Van Milligen et al., 1995; Pradhan et al., 2012) that the bispectrum measures phase coupling, defined to occur when the sum of phases between two frequencies is equal to the value of a third frequency plus a constant. Furthermore, the real and 
imaginary part of the normalized bispectrum provides information regarding how cross-frequency interactions contribute to skewness and asymmetry, respectively (for more information, see Sheremet et al., 2016, 2019a). Although it is starting to become common practice to account for asymmetry, a cnoidal wave is both symmetric and nonlinear which will cast harmonics when decomposed. Therefore, bicoherence analysis assures that all forms of distortion that result in harmonics can be accounted for (Aru et al., 2015). Notably, traditional measures of phase-amplitude coupling tend to fall short when compared to bispectral analysis (Kovach et al., 2018). For a Fourier based approach that assesses hippocampal phase amplitude coupling, we refer to our prior publication (Sheremet et al., 2019a). A thorough discussion of bicoherence and bispectral analysis, are beyond the scope of the current paper, and have been described in detail elsewhere (Dumermuth et al., 1971; Aru et al., 2015; Kovach et al., 2018).

\section{Power-power correlation}

As the hippocampal LFP transitions between quiescent activity with intermittent sharp-waves/ripples during rest and high-amplitude $\theta$ during awake-behavior, the time series at long time scales is undeniably non-stationary (that is, the mean and variance of the recorded voltage change over time). Therefore, the use of an average power spectrum over large time series has a marginalized meaning as it can average out intermittent events. To address this, a simple way to determine how the power spectra changes over time and investigate frequency interactions is to calculate the correlation coefficients across different Fourier transforms (Masimore et al., 2004, 2005) using locally stationary segments of LFP. For instance, should an increase in the $8-\mathrm{Hz}$ rhythm be accompanied by a decrease in the $12-\mathrm{Hz}$ range, a negative correlation should exist. Furthermore, if the spectrum undergoes local change (e.g., increases in the 8- $\mathrm{Hz}$ rhythm is accompanied by an increase in the adjacent $7-$ and $9-\mathrm{Hz}$ band, a non-zero width will be present (Masimore et al., 2004, 2005). Velocity is a primary driver of variance for both $\theta$ and $\gamma$ (Sheremet et al., 2019a,b). Thus, all velocities were considered together, as this approach enabled the parametric space to detect potential relationships across frequency bands.

\section{Power triggered spectral decomposition}

Stationarity describes the property of a time series in which the overall characteristics, such as the average and variance of the power, remain constant over time. It has been previously argued that slow and fast $\gamma$ rhythms are non-stationary and highly transient. However, nonstationary events, such as ripples, can be treated as "locally stationary" should it be possible to detect the event and in turn, realize the spectra (Pesaran et al., 2018). This approach allows one to discuss statistical characteristics of ripples such as the average frequency and power. Knowing that slow $\gamma$ is defined by a frequency range between 25 and $55 \mathrm{~Hz}$, and these theoretical events have been detected by instances of power that exceed 2 SDs above the mean power (Bieri et al., 2014), then it should be possible to calculate the Fourier spectra on slow $\gamma$ using this approach. The utility of detecting such transient events can be compared by running the same procedure on ripples.

Therefore, instances of high-power slow $\gamma$ events or ripple events were identified as described previously (Maurer et al., 2006; Skaggs et al., 2007). Briefly, the raw LFP was filtered either in $25-$ to $55-\mathrm{Hz}$ range (slow $\gamma$ ) or $120-$ to $250-\mathrm{Hz}$ range (ripple) and rectified by squaring the filtered trace. The mean and SD of the upper envelope of the rectified trace was calculated. For every instance in which the envelope exceeded the mean plus 2 SDs of the entire trace, $2 \mathrm{~s}$ of the raw LFP was extracted and spectrally decomposed using either Fourier decomposition with Thomson's multi-taper or wavelet decomposition.

\section{Spike frequency analysis}

Often the presence or absence of an oscillation is buttressed by whether single-unit firing is modulated at a specific frequency by examining the phase relationship between an oscillation and neuron spiking. Thus, to determine the frequency in which neuronal spiking occurred, we implemented spectral analyses on spike trains (Leung and Buzsáki, 1983; Sheremet et al., 2016). Single-unit data were generously provided by the Buzsáki laboratory and curated by the Collaborative Research in Computational Neuroscience (Mizuseki et al., 2014; Pastalkova et al., 2015; seven datasets: maze05.005, maze06.002, i01_maze06.005, i01_maze08.001, i01_maze08.004, maze13.003, and maze15.002). For these datasets, the rat performed a delayed alternation task on a figure-8 maze, running in a wheel during the delay. Only datasets in which CA1 neuron recordings were obtained bilaterally were used for the analysis. Action potentials for pyramidal cells and interneurons were initially sorted into velocity bins, analyzing the $5-15$ or $35+\mathrm{cm} / \mathrm{s}$ conditions separately. These spike times were converted into a binary time series with a sampling frequency of $1250 \mathrm{~Hz}$. As this bin size is slightly smaller than the traditional window of $1 \mathrm{~ms}$ used for waveforms, a boxcar convolution was performed such that a single spike registers as one across three adjacent bins. This output was then passed through the pmtmPH (https:// www.mathworks.com/matlabcentral/fileexchange/2927pmtmph-m) function to calculate the PSD based on multi-taper analysis. Each unit's power was normalized by the total power and then sorted by peak $\theta$ frequencies to align the harmonics between units. The average and standard error power spectra are presented.

\section{Spike-LFP coherence}

The general theory behind spike-LFP coherence is that synaptic events, which are the primary component in shaping the local-field potentials (Buzsáki et al., 2012), are responsible for generating action potentials, which in turn generate further synaptic events (Pesaran et al., 2018). Thus, if action potentials occur in support of a fundamental rhythm, then a peak in spike-field coherence may be evident even in the absence of a peak in the LFP spectrum (Pesaran et al., 2018). Therefore, using the CRCNS datasets from the Buzsáki laboratory described above, we 
calculated the spike-LFP coherence (Leung and Buzsáki, 1983) as a function of running speed.

\section{Spike preferred frequency of modulation analysis}

While spike-LFP coherence quantifies the degree to which neurons fire to a particular phase of an oscillation, in related studies, spike-LFP coupling was determined through a modulation analysis (Colgin et al., 2009; Schomburg et al., 2014). Therefore, we revisited these analyses. Using the CRCNS data, the phase of neurons was calculated relative to a narrow band filter of the LFP, in $1 \mathrm{~Hz}$ steps from $2-120 \mathrm{~Hz}$. Cells were considered to be phase locked if they differed significantly from a uniform distribution (using a $p<0.01$ as in Schomburg et al., 2014). In the instance that the cell exhibited significant phase locking, the preferred phase, and depth-ofmodulation (Skaggs et al., 1996) was calculated. For all significant cells, a mean modulation index providing the phase and vector magnitude of modulation can be realized for each frequency. Finally, this approach provides the ability to determine the fraction of cells that are significantly modulated at each frequency as well as the primary preferred frequency of each neuron. To be included in this analysis, neurons must have had an average firing rate above $0.25 \mathrm{~Hz}$.

\section{Results}

\section{Investigation of cross-frequency interactions within the hippocampus}

We have previously conducted an extensive investigation of cross-frequency interactions between $\theta$ and $\gamma$ in the hippocampus (Sheremet et al., 2019a). Using filterless approaches of power-power correlations (Masimore et al., $2004,2005)$ and phase-coupling assessed through bicoherence analysis (Sheremet et al., 2016, 2019a), we found interactions between $\theta$, harmonics, and a broad unitary $50-$ to $120-\mathrm{Hz}$ band. Notably absent in our results was the $25-$ to $55-\mathrm{Hz}$ slow $\gamma$ band. Instead, at high velocities, frequencies between 25 and $55 \mathrm{~Hz}$ were dominated by the harmonics of $\theta$. While it could be argued that slow $\gamma$ is transient to the point of being averaged out (considered below), the same transience argument has been made for the $60-$ to $100-/ 140-\mathrm{Hz}$ fast $\gamma$ rhythm (Colgin et al., 2009; Bieri et al., 2014), which was resolved by both crossfrequency analytical approaches. With this consideration that there may be something unique to our rats or data collection methodologies that preclude finding slow $\gamma$, and to enhance scientific rigor we revisited crossfrequency interactions using data from two rats generously made available from the Buzsáki laboratory (see Materials and Methods).

Using current-source density, an "electroanatomic" reconstruction of position can be conducted to identify recording location (Berényi et al., 2014). As the CA1 pyramidal layer was the site of the initial description of slow $\gamma$ (Colgin et al., 2009) as well as recent replication (LopesDos-Santos et al., 2018), the electrode in the layer was identified based on current source densities triggered to ripple events (Fig. 5A,B). In agreement with our prior publications (Sheremet et al., 2016, 2019a,b), there are multiple prominent peaks in the CA1 pyramidal layer that align with $7-10,14-20$, and $21-30 \mathrm{~Hz}$ indicative of $\theta$ harmonics (Fig. 5C). Within the $50-$ to $120-\mathrm{Hz}$ range, there was a mild increase in power with velocity (Ahmed and Mehta, 2012). While $\theta$ and the harmonics increased, the frequency bands less than $\theta$, between $\theta$ and the first harmonic and between the 18- and $27-\mathrm{Hz}$ harmonic lost power with velocity. Finally, there was a redistribution of power between the $27-\mathrm{Hz}$ harmonic and the start of the $50-$ to $120-\mathrm{Hz} \gamma$ range.

The power spectra were calculated for non-overlapping 1-s segments. By correlating the vector of power of one frequency band with another, it is possible to determine whether there is a positive or negative relationship (Masimore et al., 2004, 2005). As can be seen in Figure 5D, as $\theta$ power increases, there is a concurrent increase in $\theta$ harmonic power, as indicated by the multiple lowfrequency correlations. Furthermore, there is a correlation between $\theta$, the first harmonic $(18 \mathrm{~Hz})$ and a broad, unitary $\gamma(50-130 \mathrm{~Hz})$. Note that there is an inverse correlation between the frequencies $<9 \mathrm{~Hz}$ and the unitary $\gamma$ band, revealing that the $50-130 \mathrm{~Hz}$ increases in power while the low frequencies lose power. That is, while $\theta$ and the harmonics increased in power, the inter-harmonic intervals lost power. This redistribution of power is reflected in the positive correlation between the sub $\theta$ frequency and the $\sim 14-$ to $15-\mathrm{Hz}$ band. Finally, there is little in the way of structure between the $36 \mathrm{~Hz} \theta$ harmonic and the start of the unitary $\gamma$ band at $50 \mathrm{~Hz}$.

While this is descriptive of the LFP, it does not account for the potential effects of volume conduction. Therefore, to ensure that our observations were carried by local voltage changes, the PSD of the current source density was calculated for the pyramidal layer. Specifically, any signal that was common to the electrode above and below the pyramidal cell electrode was removed. Once again, as velocity increases, there was a redistribution of power such that $\theta$ and harmonics become more prevalent. Power also increased in the $50-$ to $120-\mathrm{Hz}$ range. Moreover, there was a loss of power in the inter-harmonic intervals (Fig. 5F). The average power correlation of the current source density reflects this observation with positive correlations between $\theta$ and its harmonics, $\theta$, and the $50-$ to $130-\mathrm{Hz}$ range and the inter-harmonic intervals. Negative correlations were notable between $\theta$ and the inter-harmonic intervals and $\gamma$ and the inter-harmonic intervals. Again, there was little structure between the last interaction indicative of a harmonics and the start of the $50-$ to $130-\mathrm{Hz}$ interaction, which is the range typically corresponding to slow $\gamma$.

Therefore, the data up to this point do not support the idea there is a slow $\gamma$ band that interacts with $\theta . \theta$ Has long been documented to increase in power with velocity (Whishaw and Vanderwolf, 1973; Morris and Hagan, 1983; Rivas et al., 1996; Shen et al., 1997; Maurer et al., 2005). Should there be any systematic change in slow $\gamma$ with velocity, it should be evident in the power correlations (Fig. 5D,F). However, as $\theta$ power increases, the slow $\gamma$ band neither increase nor decreases. It is worth noting here that the literature is obtuse regarding changes in 
A

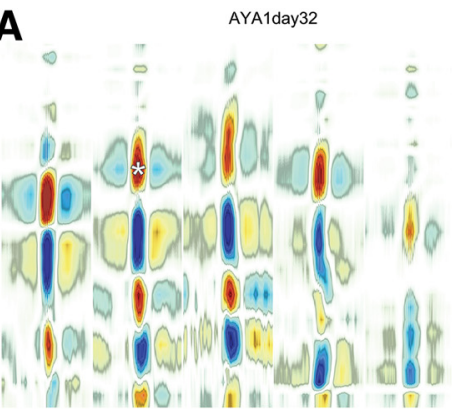

C

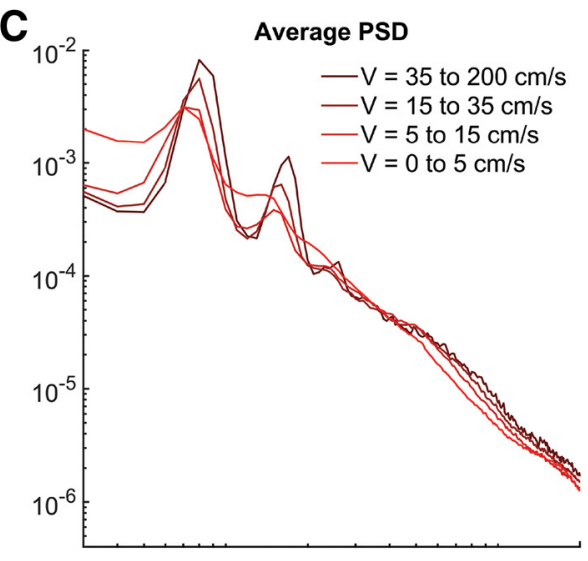

E

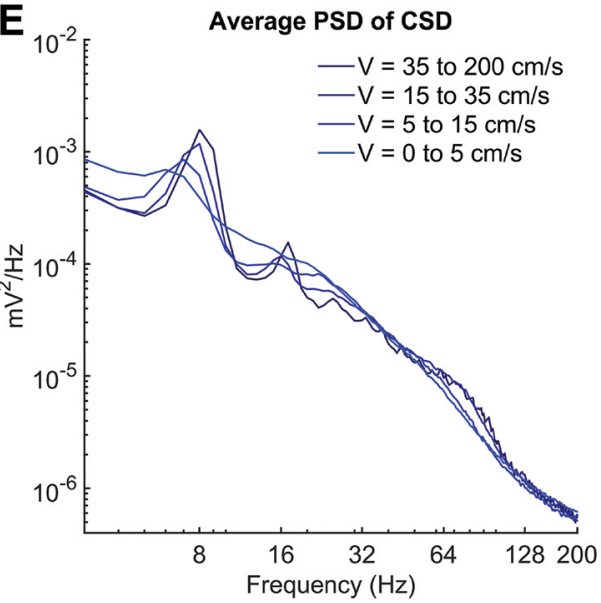

G

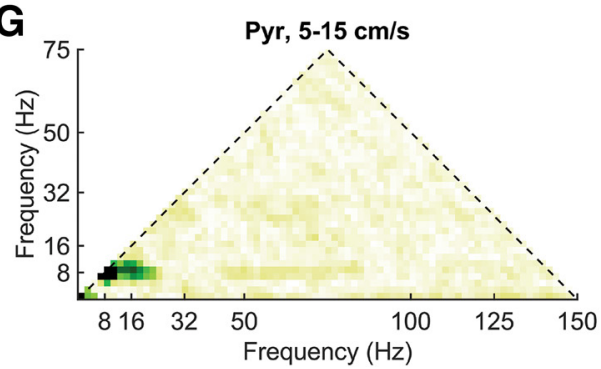

B

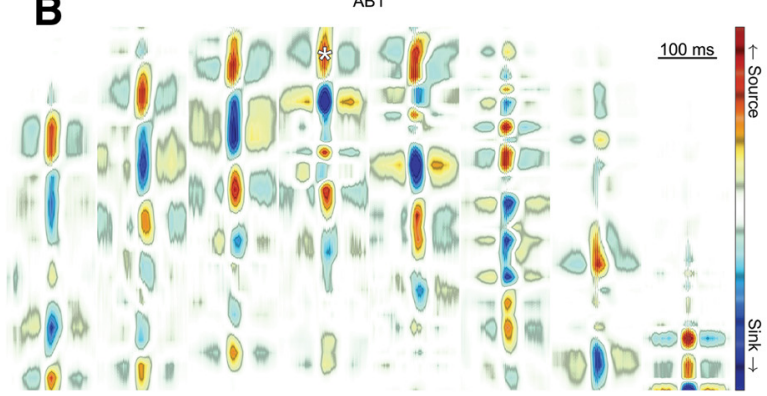

D
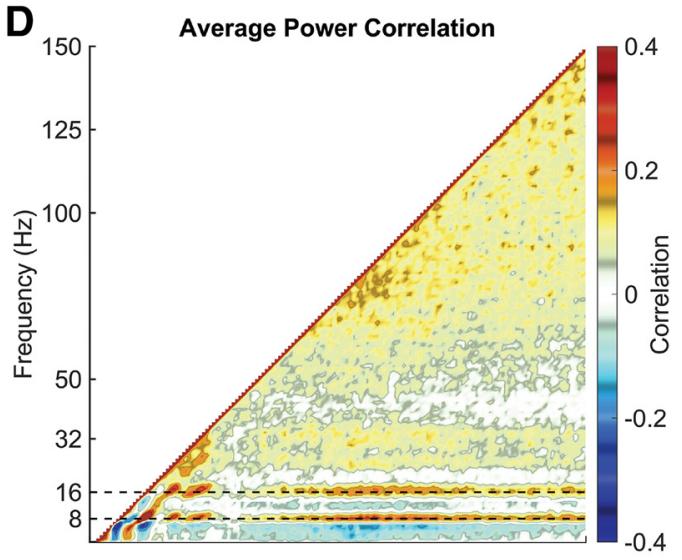

$\mathbf{F}$

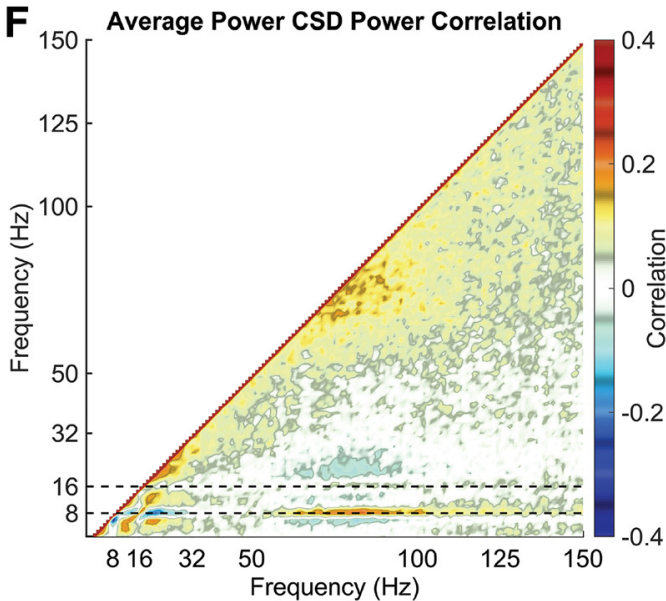

H

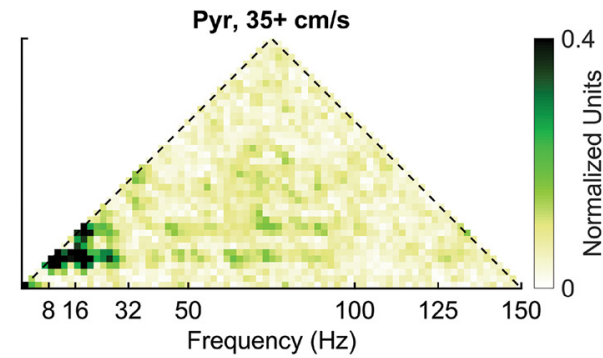

Figure 5. Cross-frequency interactions as a function of velocity. $\boldsymbol{A}, \boldsymbol{B}$, Current source density plots of local-field potential data triggered to ripples for two rats generously made available by the Buzsáki laboratory. Using the source-sink profile, layers can be easily identified. The location of the white asterisks depicts the channel location selected for each rat for the following analyses. C, Rat average CA1 pyramidal layer PSD as a function of velocity. Overall, the PSD has an inverse relationship between amplitude and frequency $\left(A=f^{-\alpha}\right)$ such that lower frequencies are higher power. As running speed increases, the power of $\theta$ as well as the peaks at 18 and $27 \mathrm{~Hz}$ increase with velocity. There is also an associated increase in the 50- to $120-\mathrm{Hz}$ range with velocity (described as a moving front; Sheremet et al., 2019b). At low frequency and within the inter-harmonic intervals, there is a loss of power. $\boldsymbol{D}$, To examine the redistribution of power across frequencies, multiple different PSD realizations were cross-correlated. Major regions of interactions include what appears to between harmonics of $\theta$ and between $\theta$ and a broad $\gamma$ band $>50 \mathrm{~Hz}$. Negative correlations are 
continued

evident between frequencies that lose power with running speed (e.g., $<6 \mathrm{~Hz}$ ) and those that increase in power with running speed (e.g., $9 \mathrm{~Hz}$ ). $\boldsymbol{E}$, In the instance that slow $\gamma$ may be overpowered by a volume conducted oscillation, the PSD was also conducted on the same CA1 pyramidal channel after calculating the current source density using the adjacent channels. The redistribution of power becomes more evident in this depiction with the inter-harmonic intervals losing power as the $\theta$ and harmonics increase in power. $\boldsymbol{F}$, The PSD correlation of the current source density trace from the CA1 layer. Note little interaction between $32-50 \mathrm{~Hz}$ and $\theta$, suggesting that this band range does not demonstrably change as a function of velocity. $\boldsymbol{G}, \boldsymbol{H}$, Average bicoherence of the CA1 LFP at low and high velocities, respectively. Note that while there is a three-wave interaction among the harmonics of $\theta$ (Sheremet et al., 2016), there is a notable dearth of interaction in the slow $\gamma$ range.

slow $\gamma$ with velocity: Zheng et al. (2015) reported a minimal change with increasing velocity, Kemere et al. (2013) reported a decrease, Chen et al. (2011) found an increase, and Ahmed and Mehta (2012) suggest that there is only one $\gamma$ band that increases in frequency and power with velocity. In light of this ambiguity, any potential result risks being construed as supporting slow $\gamma$. For instance, the power of slow $\gamma$ is not modulated by the power of $\theta$, but the interaction resides in phase coupling. Or it may be argued that the highest correlation observed near $36 \mathrm{~Hz}$ is actually slow $\gamma$.

Therefore, to address these two possibilities, we implemented bicoherence analysis to quantify the degree of cross-frequency phase coupling in the LFP (see Materials and Methods). Bicoherence is uniquely suited to identify harmonics as well as other forms of phase coupling (Aru et al., 2015; Sheremet et al., 2016). As slow $\gamma$ phase has been reported to be coupled to $\theta$ phase (Belluscio et al., 2012; Colgin, 2015a), should this be true, then an interaction should be evident.

In congruence with our prior reports, the presence of $\theta$ harmonics became more prominent with running speed across all layers (Sheremet et al., 2016, 2019a; Fig. 5G,H). There is a peak at 9 and $27 \mathrm{~Hz}$ indicating a harmonic at 36 $\mathrm{Hz}$ and a mild peak between 18 and $27 \mathrm{~Hz}$ suggestive of a $45-\mathrm{Hz}$ harmonic (for instructions on how to read a bicoherence plot, see Sheremet et al., 2016). As the power of $\theta$ harmonics increases with velocity (Sheremet et al., 2016, 2019a) and a harmonic is defined as being a phase coupled integer to a fundamental rhythm, the increase in the bicoherence between the harmonics with velocity support the interpretation that the low frequencies correlations in Figure $5 D, F$ are indeed harmonics and not slow $\gamma$.

\section{The redistribution of power in the Fourier spectrum}

As described above, power spectra undergo a redistribution of power across frequencies with velocity. $\theta$, the harmonics and the 50- to $120-\mathrm{Hz} \gamma$ band increase in power, whereas the inter-harmonic intervals decrease in power. Investigations into the relationship between slow $\gamma$ and velocity have been contradictory (Chen et al., 2011; Kemere et al., 2013; Zheng et al., 2015). As an alternative to the two $\gamma$ hypothesis, it has been argued that there is a single $\gamma$ frequency which increases with running speed, resulting in a decrease in amplitude of low-frequencies and an increase at higher frequencies (Ahmed and Mehta, 2012). As described by Ahmed and Mehta (2012), the redistribution of power across frequencies is a consequence of increasing afferent drive onto interneurons
(Traub et al., 1996). Specifically, with a low level of afferent input, neurons are weakly entrained exhibiting a range of oscillations. These same neurons become strongly entrained with energy (increasing afferent input) and velocity (Sheremet et al., 2019a). By allowing neurons to interact with each other, a general frequency of entrainment is selected, accompanied by accretion of power in one band with erosion of power in the adjacent bands (Wiener, 1965, 1966; Strogatz, 1994).

We, therefore, examined the relationship between running speed and the power spectra with higher velocity resolution. Figure 6 shows the power spectra between 0 $\mathrm{Hz}$ and $1000 \mathrm{~Hz}$ and for a narrower frequency range (25-240 Hz). Consistent with previous reports (Czurkó et al., 1999; Terrazas et al., 2005; Sheremet et al., 2016, 2019a), as running velocity increases, there is an associated increase in $\theta$ power and its harmonics. As described above, the increase in $\theta$ power and its related harmonics was associated with decreases in power in the frequency ranges surrounding $\theta$ and its harmonics. Because CA1 neurons are tuned to fire at $\theta$ frequency (see Oscillatory modulation of CA1 neuron firing), these observed increases in power at faster running speeds, which also produce higher firing rates of both pyramidal cells and interneurons (McNaughton et al., 1983), can be viewed as the energy into the hippocampus increasing resonance or bringing otherwise incoherent neural activity into coherent, dynamic patterns. A similar idea has been proposed by Traub et al. (1996), in which $\gamma$ oscillations increase in frequency with increasing drive with the only extension here being that the drive comes in bouts of $\theta$. From this perspective, it becomes odd to describe slow $\gamma$ as a fundamental oscillation that is maximal in power at low velocities. Accurately, low velocity describes the lowest firing rate condition and a minimal capability of neurons to interact on each other in a manner described by Wiener and Strogatz (Wiener, 1965, 1966; Strogatz, 1994). To consider the loss of power to be data that supports a slow $\gamma$ rhythm is contradictory as the low velocity/low afferent input state implies that neurons are free to "drift," and by lacking entrainment with each other, have limited coherence to the LFP. Given this rationale, we explored the frequency of spike modulation, spike-LFP coherence analysis, and a phase-locking ("depth of modulation") analysis as a function of velocity.

\section{Oscillatory modulation of CA1 neuron firing}

Prior analyses of hippocampal neuron spike times have described modulation by slow $\gamma$ (Colgin et al., 2009). 
A

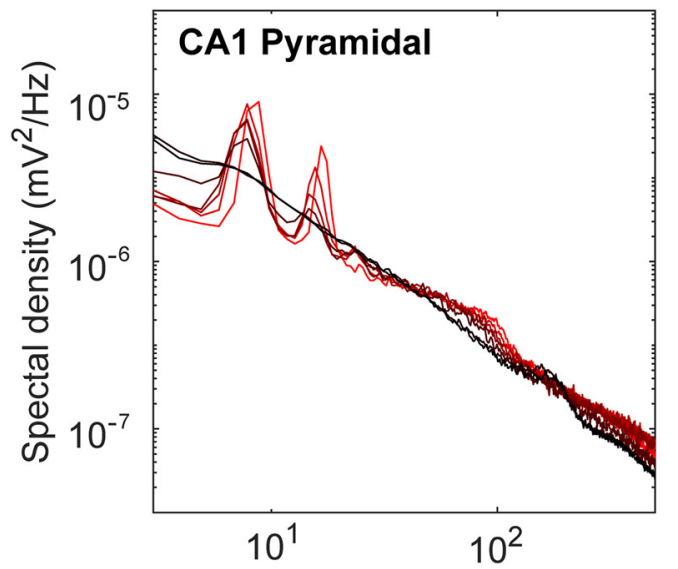

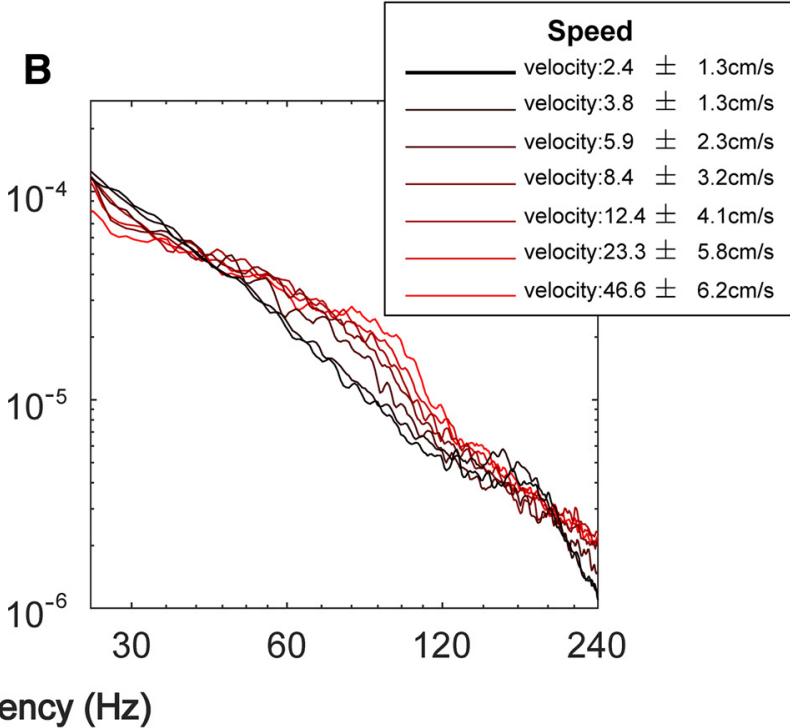

Figure 6. Spectral changes with velocity. A, The PSD across low to high velocities for frequency ranges up to $500 \mathrm{~Hz}$. The power of $\theta$ and its associated harmonics increases with faster running velocities. This increase in $\theta$ and harmonic power is associated with a decrease in the power in the inter- $\theta$ /harmonic frequency bands at higher running speeds. $\boldsymbol{B}$, The PSD across the $25-$ to $240-\mathrm{Hz}$ frequency ranges. At higher velocities, power in the 25-50 range decreases. Compare to Ahmed and Mehta (2012; Fig. 2).

When examining the preferred phase of the firing of neurons to the hippocampal $\theta$ rhythm, however, the spike depth of modulation plots are not pure sinusoids. Rather, there is a definite skew and asymmetry (Skaggs et al., 1996; Quilichini et al., 2010). Furthermore, spectral decomposition of spike trains has revealed the presence of a $\sim 16-\mathrm{Hz}$ harmonic modulation (Sheremet et al., 2016). In light of the skewness, the concern has been raised that modulation in the $20-$ to $30-\mathrm{Hz}$ band may not actually be related to slow $\gamma$ but rather coupled to the asymmetry of $\theta$ (Schomburg et al., 2014). Therefore, we compared the phase coupling of neurons to slow $\gamma$ versus higher-order $\theta$ harmonics in the LFP in relation to the animal's running speed.

First, we replicated the analysis of Schomburg et al. (2014), determining the preferred frequency of modulation as well as the proportion of neurons modulated across frequencies (Fig. 7). The only major difference between our approach and that of Schomburg et al. (2014) is that our phase assignment was not conducted via wavelet but through phase assignment relative to a narrow band filter. When considering all frequencies between 4 and $128 \mathrm{~Hz}$, it is evident that most cells exhibit their strongest modulation relative to the $7-$ to $9-\mathrm{Hz} \theta$ rhythm (Fig. 7A). Over $90 \%$ of interneurons preferred $\theta$ at high velocities. Including pyramidal cells, it can be safely stated that nearly every hippocampal neuron is first and foremost modulated by $\theta$. As $\theta$ preference eclipses all other frequencies, the explicit consequence is that -during behavior- any other frequency modulation is secondary. Therefore, to explore potential "secondary preferred frequencies," the same analysis was performed for oscillations $>20 \mathrm{~Hz}$ (Fig. $7 B$ ). A small clustering is evident in the $21-$ to $27-\mathrm{Hz}$ range (although the proportion is much lower than Fig. 7A). Parsimoniously this can be explained by harmonic effects: (1) most neurons are strongly modulated by $\theta$ (Fig. 7A), (2) depth of modulation plots of neurons relative to $\theta$ are asymmetric (Skaggs et al., 1996; Quilichini et al., 2010), and (3) harmonic modulation can carry this effect (Schomburg et al., 2014). This approach, however, is "either/or," not explicitly considering the proportion of the population that is modulated by all frequencies. When asking the question from this angle (Fig. $7 C$ ), as anticipated, the maximum peak was observed at $\theta$ followed by the 14- to $18-\mathrm{Hz}$ harmonic and the $21-$ to $27-\mathrm{Hz}$ harmonic (interneuron; high velocity). While these values are indicative of significant phase coupling, the average depth of modulation describes how tightly coupled the neuron spikes are to a specific phase (Skaggs et al., 1996; Fig. 7D). In the pyramidal cells, low firing rates at low velocity skew the depth of modulation to an artificially high level (that is, bins with a single spike at the trough will cause artificial inflation). With that consideration, the largest depth of modulation resides at $\theta$ at both low and high velocity for pyramidal neurons. The interneurons exhibit a similar pattern with the additional peak at the first harmonic of $\theta$ at high velocity. These values steadily decrease from $\theta$ toward higher frequencies, approaching an asymptotic floor near 32-64 Hz (slow $\gamma$ ).

Finally, as the depth of modulation analysis provides the preferred phase of firing as well as the strength of the modulation, we calculated the average preferred vector of firing for neurons that were significant (as determined by a Rayleigh test $p<0.01$ ) as a function of velocity (Fig. 7E). As anticipated from Figure $7 D$, the magnitude of modulation was larger at low velocities, although with little consistency in adjacent frequencies. As running speed increases, adjacent frequencies tend to have similar phase preferences. Note well that almost every neuron was modulated by $\theta$, making any other frequency modulation secondary. With respect to other frequencies, as 
A Maximum Preferred Freq.
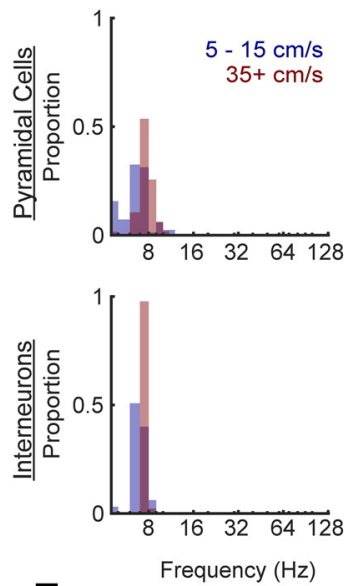

E

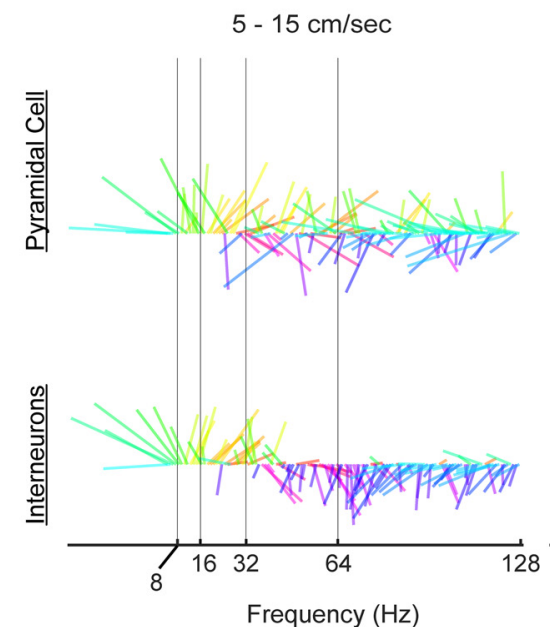

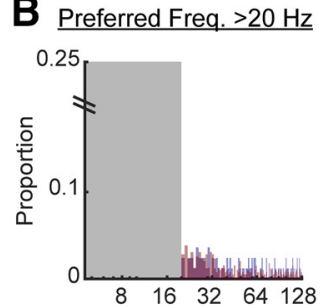

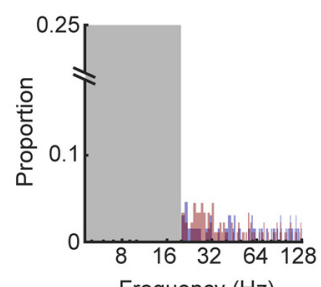

Frequency $(\mathrm{Hz})$
C Significant Phase Locking
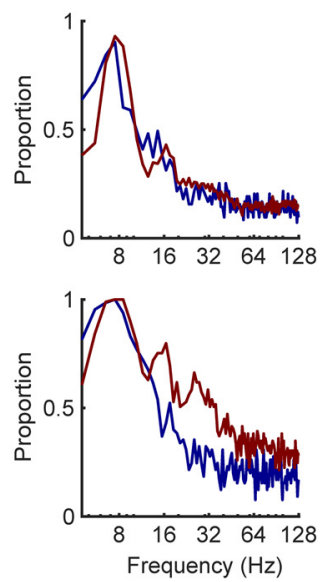

D Average Depth of Modulation
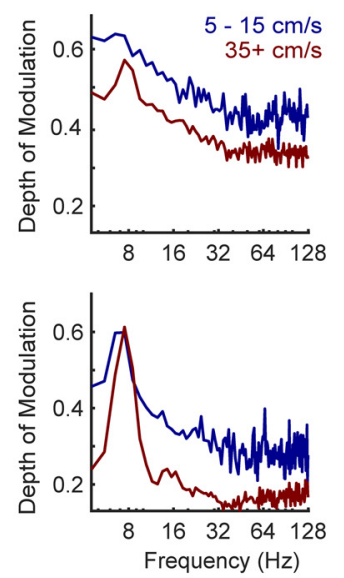

$35+\mathrm{cm} / \mathrm{sec}$

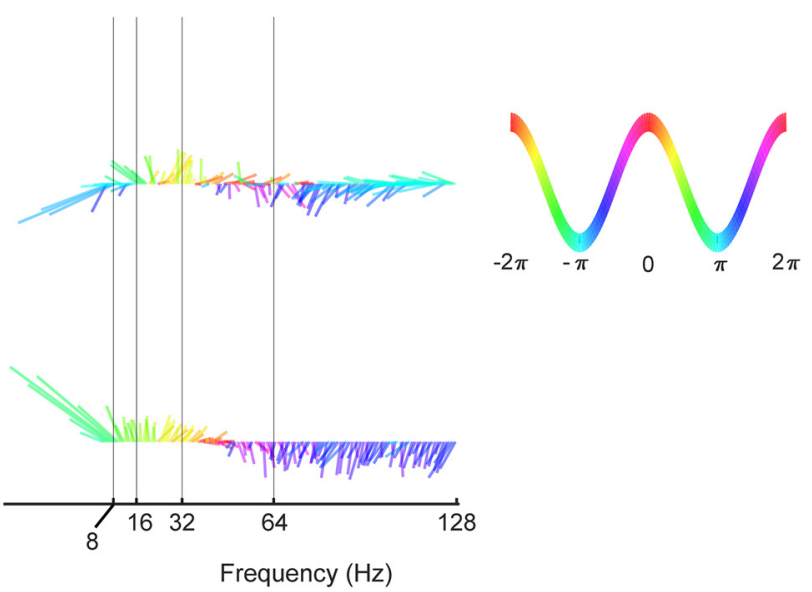

Figure 7. Neuronal depth of modulation can be found for all frequency bands but with little evidence for a slow $\gamma$ band. $\boldsymbol{A}$, The maximum preferred phase of neuron modulation was determined for all cells should the neuron have a significant phase locking (as determined by a Rayleigh test $p<0.01)$. When conducting this analysis across a broad band $(4-128 \mathrm{~Hz})$ and as a function of velocity, it becomes evident that neurons are primarily modulated by $\theta$. $\boldsymbol{B}$, However, $\gamma$ modulation analyses are often conducted without considering the contribution of $\theta$. Therefore, we examined the maximum preferred phase $>20 \mathrm{~Hz}$. The major mass of spikes falls near the third harmonic of $\theta$, consistent with the heavy $\theta$ modulation seen in panel $\boldsymbol{A}$ as well as hypothesized by Schomburg et al. (2014). Note the difference in $y$-axes between panels $\boldsymbol{A}, \boldsymbol{B}$. $\boldsymbol{C}$, Similar to Schomburg et al. (2014), the proportion of cells that exhibited significant phase locking (as determined by a Rayleigh test $p<0.01$ ) was plotted as a function of frequency. However, the primary difference between Schomburg et al. (2014), and the present study was that the aforementioned study implemented wavelet to determine phase, whereas the current study used a narrow band filter (see Materials and Methods). Note that the majority of cells were modulated by the 7- to $9-\mathrm{Hz} \theta$ rhythm. Should they exhibit a skewed distribution in their phase preference (Skaggs et al., 1996), then it is conceivable that they would also exhibit significant phase locking to harmonics. $\boldsymbol{D}$, For each neuron that exhibited significant phase locking to a specific band, the depth of modulation was calculated. In this instance, the sparse firing of pyramidal neurons favors greater depths of modulation at lower velocities (as depth of modulation is the maximum bin value minus the minimum bin value normalized to the maximum; see Materials and Methods). At both low and high velocities, for pyramidal cells and interneurons, the maximum depth of modulation was to the $\theta$. Although there was a large proportion of interneurons modulated by the $21-$ to $27-\mathrm{Hz}$ harmonic of $\theta(\boldsymbol{C}$, bottom), the depth of modulation values in this range approach a minimum, hitting the lowest values in the slow $\gamma$ range. $\boldsymbol{E}$, For the significantly modulated neurons, a vector length (depth of modulation) and angle (preferred phase) was determined to allow the calculation of the average modulation vector as a function of frequency. The color and angle depict the phase (see key) while the magnitude of the line is the depth of modulation. At low velocities, there is a high degree of variance across adjacent frequencies which becomes smaller at higher velocities. For of all these plots, while it may be tempting to derive a conclusion for frequencies $>16 \mathrm{~Hz}$, we emphasize caution. After the $\theta$ range, spike-field coherence values fall well below 0.05 (weak coherence, despite being significant, is closer to an incoherent, random phase assignment than coherence; Fig. 8). Therefore, although it is possible to find significant coupling across the range of bands between 4 and $128 \mathrm{~Hz}$, there is no rationale to suggest that a slow $\gamma$ modulation exists in the spiking activity of the units. 
only the neurons that passed a Rayleigh test went into this analysis, it demonstrates that depth of modulation within a singular frequency band is not sufficient evidence in support of a fundamental rhythm. One can always find a significant proportion of neurons modulated to a narrow band. Concerning slow $\gamma$, modulation to $25-50 \mathrm{~Hz}$ may be a consequence of aliasing and/or coupling to $\theta$ harmonics (Schomburg et al., 2014; Sheremet et al., 2016).

This analysis, however, may not be comprehensive enough to dismiss slow $\gamma$ modulation. Therefore, we considered the alternative approaches of spike PSD and spike-LFP coherence. First, the point-process spike trains (available on the CRCNS.org website; see Materials and Methods) were sorted into either a low or high-velocity bin, based on the running speed of the rat, and converted into a binary time series. These spike trains were spectrally decomposed to examine the burst frequency modulation (Leung and Buzsáki, 1983; Sheremet et al., 2016). "Power" in this analysis is related to the neurons firing rate which, in the hippocampus follows a logarithmic distribution across the population (Mizuseki and Buzsáki, 2013). Therefore, to ensure that a high firing rate neuron does not skew the overall results, each neuron was normalized by overall power (resulting in power being presented in arbitrary units; Fig. 8). As Bieri et al. (2014) found the most slow $\gamma$ epochs in the center of a linear track, coinciding with locations of the highest running speed, it may be anticipated that there would be a single peak in the slow $\gamma$ range of the spike spectrograms at high velocity. Investigations of the spectrograms, however, do not support this idea. Instead, the small observable peaks tend to coincide with $\theta$ and the first harmonic. There is no evidence of a slow $\gamma$ modulation in the neuron spike trains for either interneurons or pyramidal cells. Notably, interneuron firing appeared to be modulated within the $50-$ to $90-\mathrm{Hz}$ range (Fig. $8 A, B$ ). Finally, we considered that, despite the absence of a depth of modulation and the absence of spikes bursting at slow $\gamma$ frequency, there might be spike-LFP coherence indicative of an oscillation (Pesaran et al., 2018). Therefore, we calculated spike-LFP coherence by neuron type and as a function of velocity (Fig. 8C,D).

Coupling was only evident in the $\theta$ and harmonic bands. However, as the Spike-LFP coherence falls below 0.05 at frequencies $>18 \mathrm{~Hz}$, we emphasize caution as these values lean toward "incoherence" (see Buzsáki and Schomburg, 2015).

Again, there was little in the way that would suggest that slow $\gamma$ exists. These results beg the question that, if slow $\gamma$ is absent in the Fourier decomposition, what is responsible for the description becoming dogma? As the initial description of slow $\gamma$ was predicated on wavelet decomposition, it is tenable that the different methods yield different decompositions with the proposition that one is not maintaining fidelity with the underlying biology.

\section{Comparison of Fourier, wavelet decomposition, and EEMD}

Fourier analysis operates by decomposing a time series into a sum of sine wave oscillations each with a fixed amplitude. There is a fixed decimation in time and frequency resolution in this approach (Fig. 2). A wavelet, on the other hand, has a trade-off between time and frequency such that low frequencies will have a low temporal, high-frequency precision. As the mother wavelet is compressed for higher temporal resolution, it comes at the sacrifice of frequency resolution (Fig. 4). A nonsinusoidal oscillation such as $\theta$, a skewed and asymmetric oscillation at $\sim 8 \mathrm{~Hz}$, would be represented in the Fourier domain as an $8-\mathrm{Hz}$ oscillation plus the phase locked, integer related harmonics to the fundamental. That is, for the superposition of sine waves to be recombined in a way that reconstructs a non-sinusoidal time series requires the use of harmonics. A wavelet, while also capable of decomposing the same time series, will treat nonsinusoidal oscillations differently. Consider the initial report of slow $\gamma$ in which the mother Morlet wavelet was set to have a ratio of 7 between the central frequency of wavelet and SD of the applied Gaussian window (Colgin et al., 2009). This setting, based on the Heisenberg timefrequency resolution boxes, is theoretically capable of resolving $\theta$ with high-frequency resolution but low temporal precision. For the superposition of wavelets to recreate a nonlinear time series (such as a sawtooth wave), the same method is subject to assigning a high amount of power across a broad range of frequencies in a transient manner. This places the wavelet decomposition method at risk of distorting the frequency-power representation of a non-sinusoidal time series. The $24 \mathrm{~Hz}$ and other highorder harmonics of $\theta$ would erroneously be decimated into Heisenberg boxes with lower frequency resolution and higher temporal resolution. The consequence being that the $24 \mathrm{~Hz}, 32 \mathrm{~Hz}$, and higher harmonics of $\theta$ could be depicted as a broadband $25-$ to $50-\mathrm{Hz}$ oscillation. In addition to wavelet analysis, EEMD has been put forward as a method theoretically capable of breaking the LFP into $\theta$ and supra- $\theta$ bands (Lopes-Dos-Santos et al., 2018). Specifically, Lopes-Dos-Santos and colleagues claim that, following EEMD, the remaining supra- $\theta$ signal is "harmonic free."

Validity describes the ability of a test or procedure to measure what it claims to measure. As each spectral decomposition method asserts to be capable of decomposing a time series into a frequency-power representation, a simple test is to evaluate their outcomes against a synthetic trace with known frequencies and powers. Therefore, an initial benchmark comparison of Fourier decomposition versus wavelet decomposition and EEMD was conducted using a synthetic time series of pink noise embedded with an oscillation at $8 \mathrm{~Hz}$ and harmonics at 16, 24, and $32 \mathrm{~Hz}$ (Fig. 9A-D). The first synthetic trace was generated by the superposition of harmonics to create a saw-tooth wave while harmonics in the second trace summed to make a completely symmetric, cnoidal wave (demonstrating that simple asymmetry calculations are insufficient to account for harmonics). Notably, the power 
A
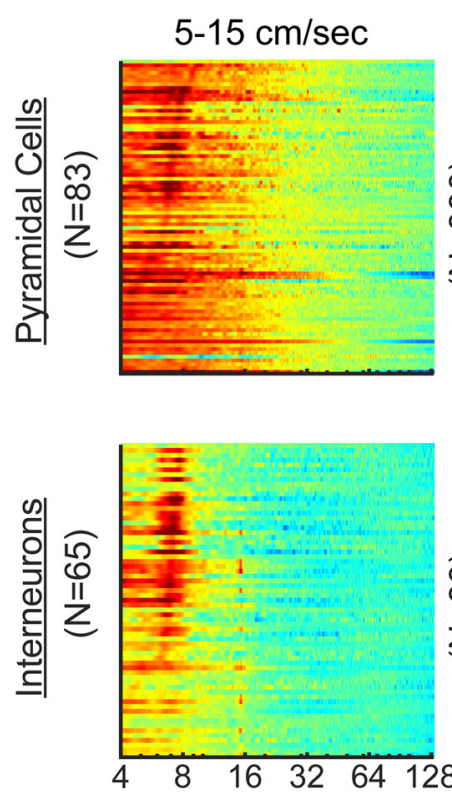

Frequency $(\mathrm{Hz})$

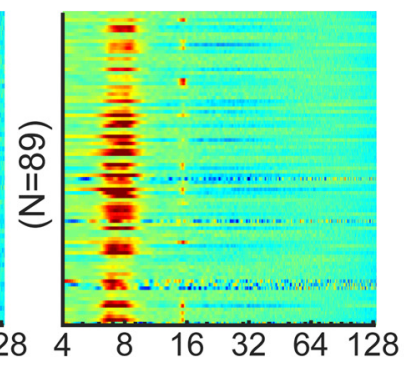

Frequency $(\mathrm{Hz})$

\section{Spike-LFP Coherence by Running Speed} $5-15 \mathrm{~cm} / \mathrm{sec}$
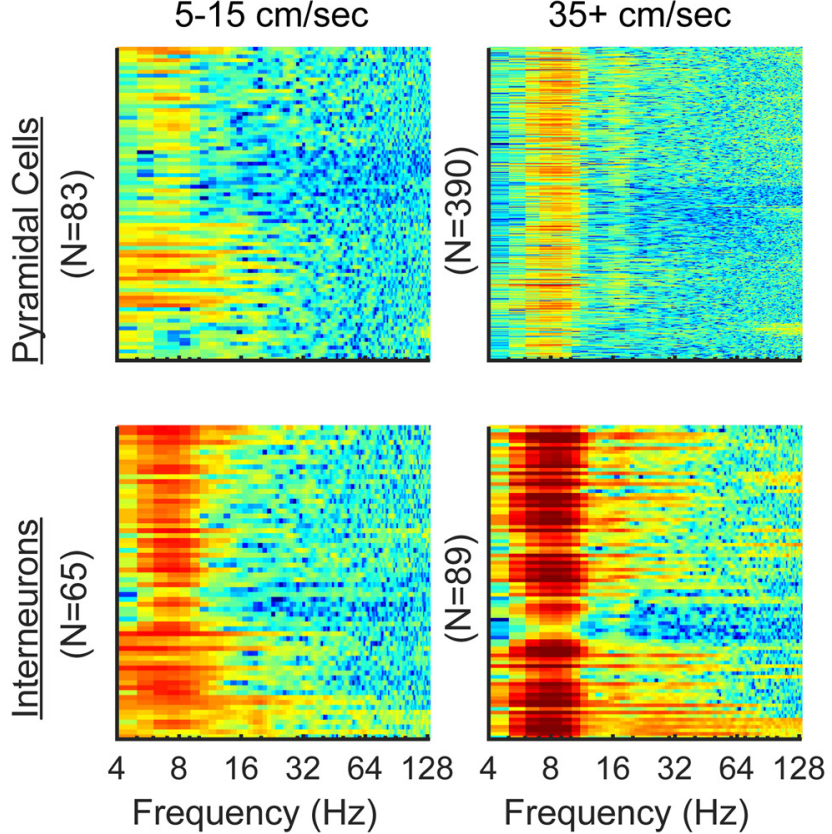

\section{B Average PSDs}
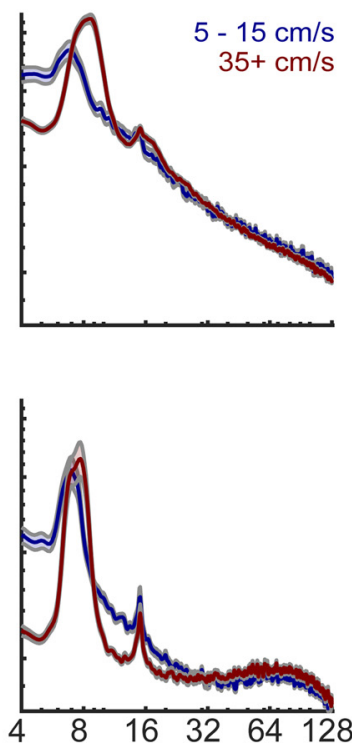

Frequency $(\mathrm{Hz})$

\section{Average Coherence}

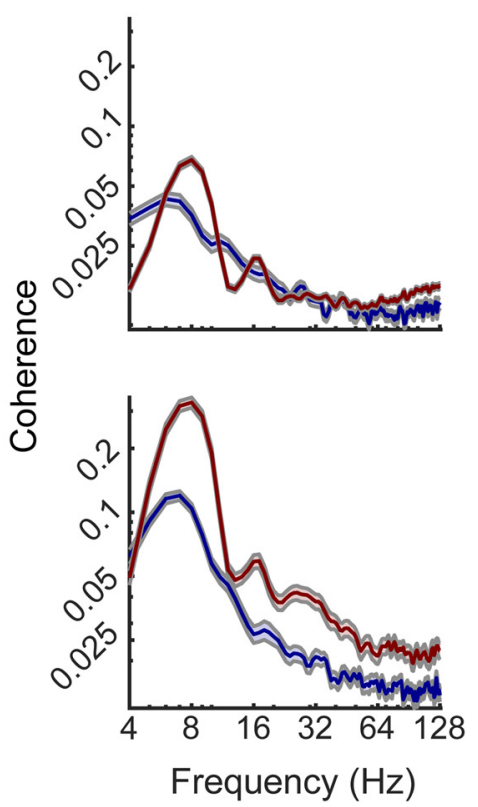

Figure 8. PSD of neuron spike trains and spike-LFP coherence as a function of velocity. $\boldsymbol{A}$, Individual power spectra for spike trains at low velocity $(5-15 \mathrm{~cm} / \mathrm{s})$ and high running speed $(35+\mathrm{cm} / \mathrm{s}$; color axes are to the same scale between plot) for interneurons and pyramidal cells. A spike threshold $>0.25 \mathrm{~Hz}$ was applied to each running bin to ensure that only neurons with a considerable number of spikes were analyzed. The rows are sorted by the maximum frequency between 4 and $12 \mathrm{~Hz}$. $\boldsymbol{B}$, The average PSDs at low (blue) and high velocities (red); error bars are the SEM (different axes are used to allow the spectral shape to be compared). Note that there is an absence of a spike-frequency peak in the slow $\gamma$ range although both the interneurons and pyramidal cells exhibit harmonic modulation. Moreover, at high velocity, there is a potential peak in the interneurons that coincides with the traditional $\gamma$ range of $40-100 \mathrm{~Hz}$ (Bragin et al., 1995). Note that the axes are in arbitrary units as power in this circumstance is determined by the number of spikes and thus, each trace was normalized by total power. $\boldsymbol{C}$, Individual spike-LFP coherence plots for interneurons and pyramidal cells at different running speeds. Once again, the color axis is equal across plots. $\boldsymbol{D}$, Average spike-LFP coherence by cell type as a function of velocity. While there is a notable difference in overall coherence as a function of velocity, the only peak are at $\theta$, the 14to $18-\mathrm{Hz}$ harmonic and the $21-$ to $27-\mathrm{Hz}$ harmonic (prominent at high velocity in the interneurons). Broad modulation in the $25-$ to $50-\mathrm{Hz}$ band (slow $\gamma$ ) is absent. We emphasize strong caution in interpreting small coherence values (for a similar opinion, also see Buzsáki and Schomburg, 2015). A coherence of 1 would indicate no variance in the phase difference across signals, while 0 would 
continued

be indicative of a random phase assignment. As the majority of coherence values related to frequencies greater than the 16- $\mathrm{Hz} \theta$ harmonic fall below 0.05 , concluding that units are capable of organizing, or being organized into higher frequencies based on this data are dangerous.

estimate of the Fourier decomposition closely matched the theoretical distribution. However, as a consequence of the multi-resolution analysis of wavelet decomposition, the power estimate significantly expands the frequency representation of the harmonics to cover a wide band, overlapping with reports of slow $\gamma$ (Colgin et al., 2009) as well as $\beta(15-30 \mathrm{~Hz}$; Rangel et al., 2015, 2016). Although harmonics were implemented in the construction of these time series, EEMD expressed the same peaks as wavelet demonstrating a failure to remove these components. The three decomposition methods were then applied to the analysis of hippocampal LFP from the CA1 pyramidal cell layer (Fig. 9E,F). Again, both the wavelet decomposition and EEMD give the impression of convolved harmonics. This is despite the claim that EEMD is free of harmonic effects (Lopes-Dos-Santos et al., 2018). To explore the degree to which EEMD accounts for the harmonics, both the synthetic trace from Figure $9 A$ and the LFP data from rat 782 (Fig. 9E) were processed with EEMD, and the resulting trace run through bicoherence, capable of detecting harmonics (Aru et al., 2015). The results of Figure 10 reveal multiple interactions at integers of $\theta$. As phaselocked integer oscillations define harmonics, this analysis demonstrates the incapability of EEMD for generating a harmonic free LFP challenging the validity of the initial claim. Furthermore, the implementation of wavelet with a ratio of 7 between the central frequency of wavelet and SD of the applied Gaussian window (Colgin et al., 2009) "convolves" over the higher-order harmonics (plausibly accounting for why studies using wavelet decomposition rarely identify a harmonic above $16 \mathrm{~Hz}$ ). Importantly, this convolution loses fidelity to the underlying oscillation gen-
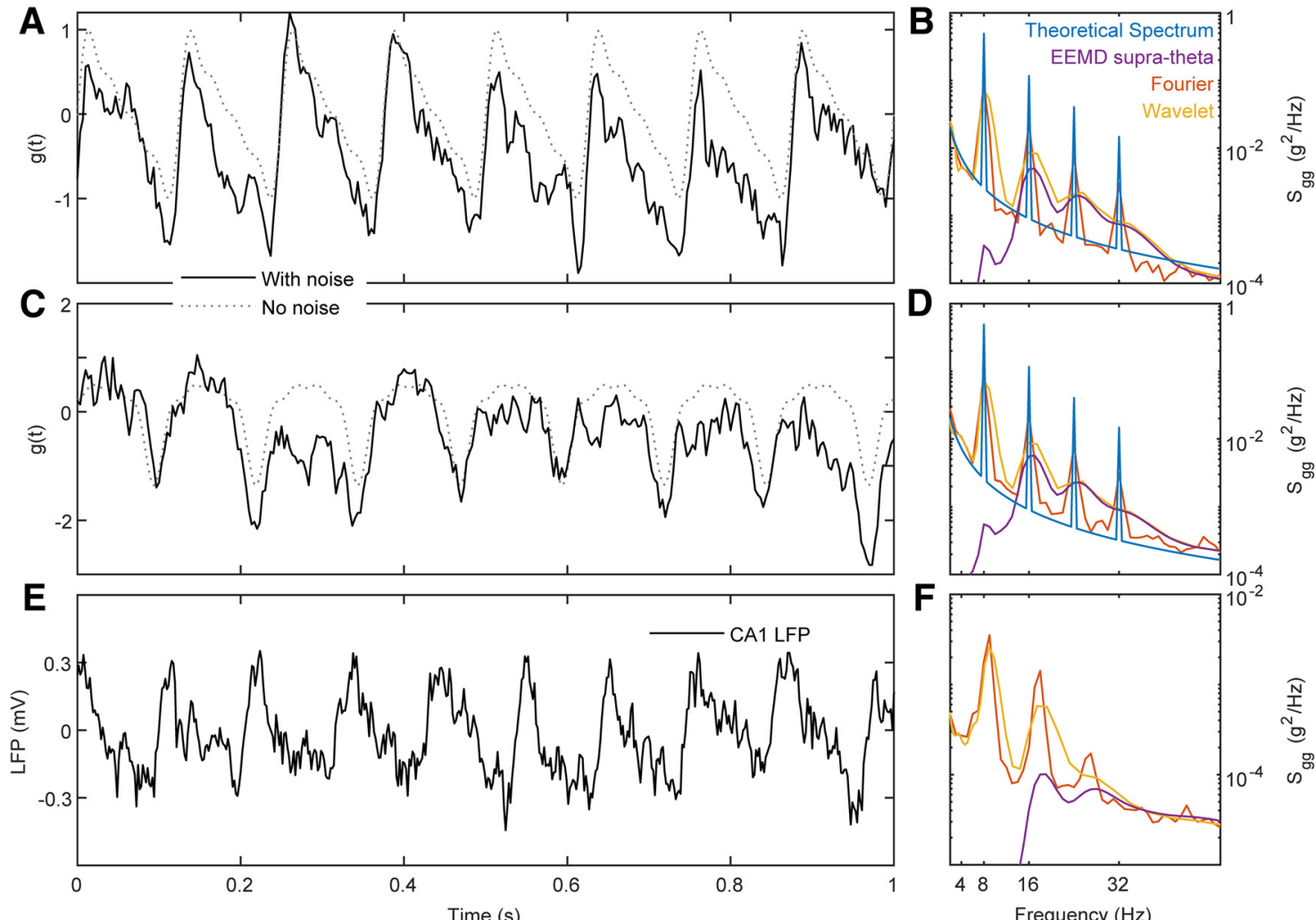

Figure 9. Example of Fourier, wavelet and EEMD analysis on synthetic time series and hippocampal LFP. A, A synthetic time series emulating an LFP recording dominated by an asymmetric $\theta$ signal. The signal is constructed from an asymmetric sine function (dashed line; an $8-\mathrm{Hz}$ fundamental oscillation with phase-coupled harmonics), and pink $\left(f^{-1.5}\right)$ noise (solid line). The inclusion of pink noise provides a more realistic spectrum for comparison to the raw LFP. $\boldsymbol{B}$, Estimates of PSD using different methods. $\boldsymbol{C}, \boldsymbol{D}, \mathrm{A}$ synthetic time series and spectral decomposition as in $\boldsymbol{A}, \boldsymbol{B}$ but with a different alignment, generating a cnoidal wave. $\boldsymbol{E}, \boldsymbol{F}$, Actual LFP from the CA1 pyramidal layer and the respective spectral decomposition. As anticipated from the spectral leakage inherent in wavelet decomposition (Fig. 4), neither the wavelet nor EEMD approaches are capable of accounting for higher order harmonics larger than $16 \mathrm{~Hz}$. Rather, the method artificially distributes power over a wide band giving the erroneous impression of a slow $\gamma$ when there is none in the underlying signal. Once again, we remind the reader that, for wavelet and EEMD, the assignment of variance to any specific frequency is ultimately arbitrary and meaningless. 

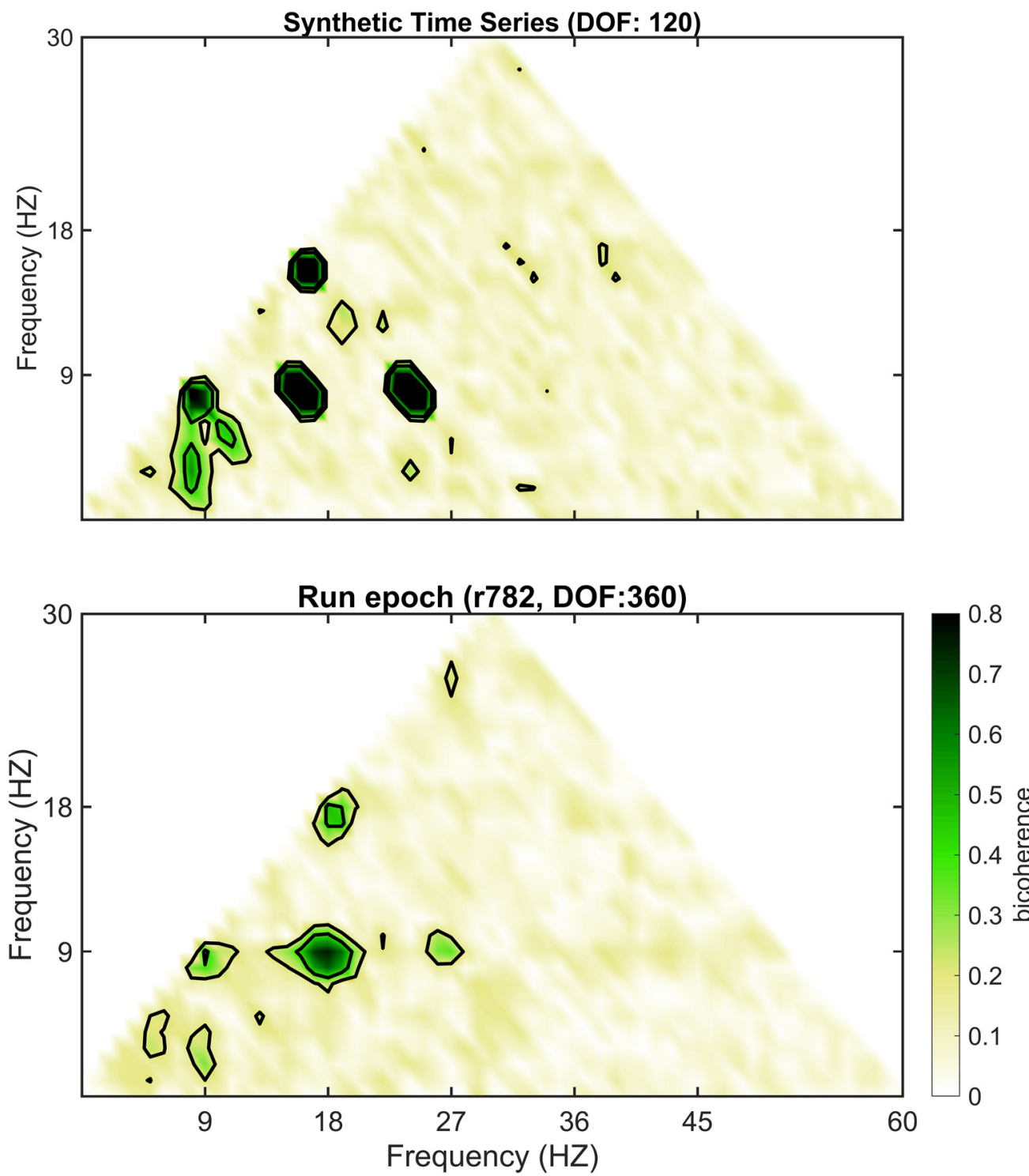

Figure 10. Post-EEMD bicoherence plots demonstrating failure to account for harmonics. In a prior study, it was suggested that "EEMD also provides supra- $\theta$ components that are virtually free from harmonic artifacts (Wu and Huang, 2009). .." (Lopes-DosSantos et al., 2018, their supplemental p. e3). However, the parent study of Wu and Huang provide no evidence testing the validity of this claim. Therefore, using a synthetic trace as well as LFP data, the post-EEMD processed time series were analyzed with bicoherence. Note that, in both instances, the contour lines outline four distinct regions of triad phase correlations ([8, 8, 16 Hz], [16, 8, $24 \mathrm{~Hz}],[24,8,32 \mathrm{~Hz}]$, and [16, 16, $32 \mathrm{~Hz}]$ ), demonstrative of strong harmonics.

erating an invalid power-frequency plot, that is, does not match the true relationship between frequency and power in the synthetic trace.

With this concern, we proceeded to revisit the wavelet scalogram to investigate the interaction between $\gamma$ frequency and the $\theta$ oscillation (Colgin et al., 2009) as well as determine whether a more reasonable decomposition could be achieved with wavelet. This was first conducted for synthetically generated data (a sawtooth wave and an oscillation with integer locked harmonics) as well as data collected from CA1 pyramidal cell layer (Fig. 11). Often understated is that the selection of Morlet wavelet parameters will dramatically alter the decomposition. The mother Morlet wavelet is characterized as the frequency of a carrier oscillation and the deviation of the Gaussian envelope. This parameter effectively alters the trade-off between the time-frequency resolution. Therefore, we also investigated the ability of each Morlet ratio to resolve the underlying frequencies in relation to time. For the scalogram decomposition of the sawtooth wave, the low Morlet wavelet ratios (4 and 7) capture the instantaneous change in amplitude by distributing power across a wide frequency band. The higher Morlet wavelet ratio (30) effectively does the reverse, capturing the nonlinear oscillation as a series of harmonic oscillations that are well resolved in frequency. While this is a simulation to an extreme derivative, the time-frequency representation of the synthetic harmonic series (8, 16, 24, 32, and $40 \mathrm{~Hz}$ ), generating a mild sawtooth wave is nearly identical to the initial report of slow $\gamma$ in the CA1 pyramidal cell layer for 

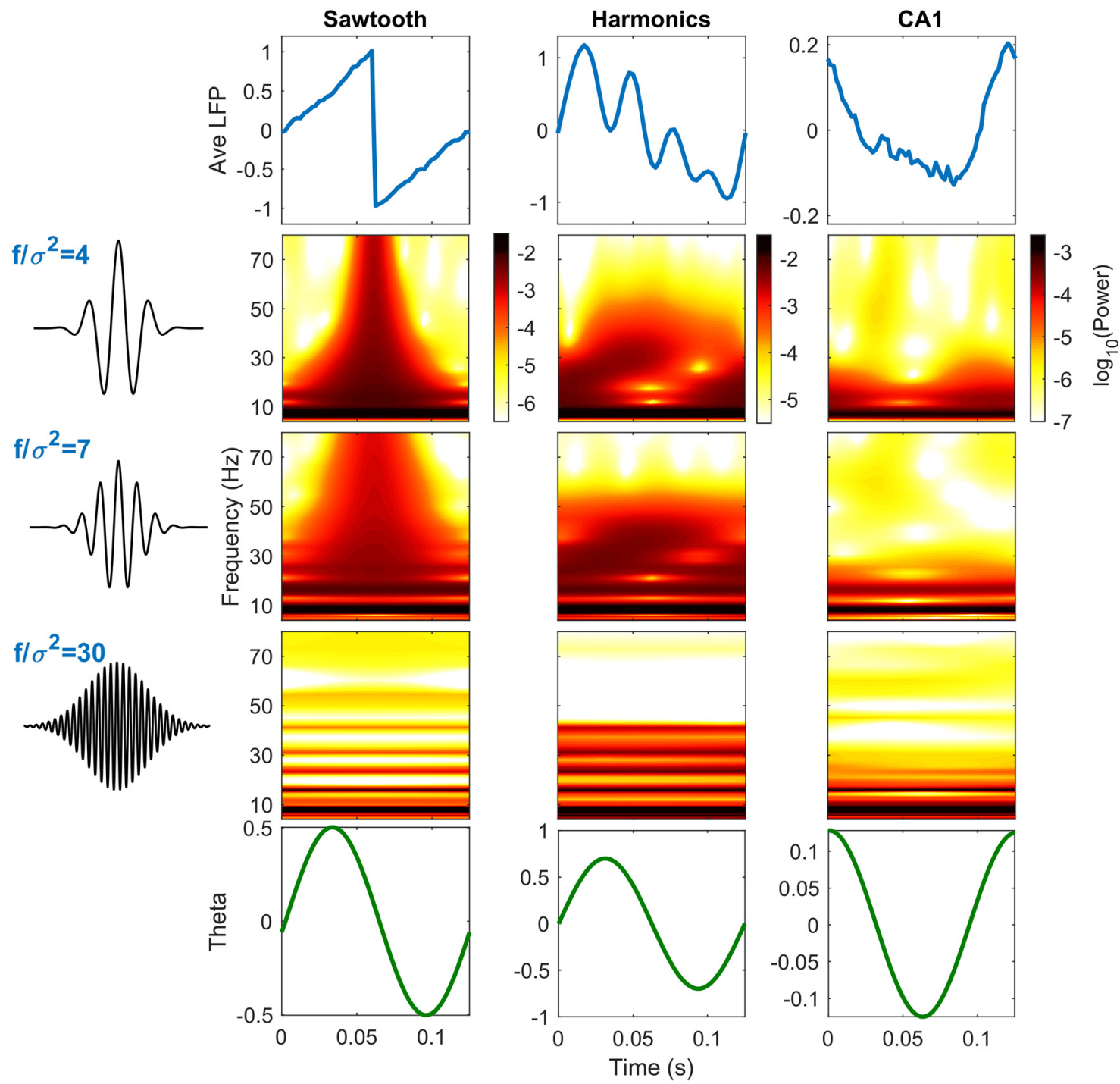

Figure 11. Scalograms for synthetic time series and LFP recordings in the CA1 pyramidal cell layer using different wavelet parameters. The averaged time series for one $\theta$ period $(8 \mathrm{~Hz})$ and wavelet scalograms were obtained for three time series: (1) synthetic sawtooth waves (left to right, the 1st column); (2) time series with fundamental $8-\mathrm{Hz}$ oscillation and its harmonics (16, 24, 32, and $40 \mathrm{~Hz}$; the 2nd column); (3) LFP recorded in CA1 pyramidal cell layer (the 3rd column). Synthetic time series have a total length of $10 \mathrm{~s}$ and were added with a pink background noise. LFP recordings were selected during high speed running with a total length of $30 \mathrm{~s}$. The averaged time series were obtained by averaging over $\theta$ periods (top row). The wavelet scalograms for averaged time series were computed with three different wavelet transforms (the three middle rows). The Morlet wavelet family is characterized by the constant ratio between the central frequency of wavelet 2 SD of the applied Gaussian window. Therefore, the results for three different Morlet wavelets are presented. Averaged time series were filtered within $\theta$ band $(7.5-8.5 \mathrm{~Hz})$ which represent the $\theta$ phase (bottom row). A sawtooth wave is decimated into an infinite series of harmonics in Fourier decomposition (https://en.wikipedia.org/wiki/Sawtooth_wave). The lowest ratio of 4 optimizes for temporal resolution over frequency resolution, whereas the highest ratio of 30 has an increased frequency resolution at the expense of temporal resolution with results that are more in line with Fourier analysis. This is depicted in the sawtooth scalograms as a temporally localized, wide frequency band event in the Morlet ratio of 4 but as frequency specific, time diffuse bands in the Morlet ratio of 30 condition. Note that the latter condition resolves harmonics. This effect is also evident when combining an $8-\mathrm{Hz}$ oscillation with its first few harmonics $(16,24,32$, and $40 \mathrm{~Hz})$ through additive synthesis in developing a sawtooth wave. The Morlet ratio of 4 convolves over the higher-order harmonics, giving the impression of a 25- to $50-\mathrm{Hz}$ cross-frequency interaction, whereas the wider ratio of 30 resolves each individual component. These effects carry over to LFP data collected from the CA1 pyramidal layer. The middle ratio of 7 was selected to match the methods of Colgin et al. (2009). Note that the parameters for the ratio of 7 are more closely aligned with the ratio of 4 in that the harmonics are heavily convolved across a wide frequency band, giving the impression of a wideband oscillation when none exists.

the low ratio wavelets (Colgin et al., 2009). Note that in this simulated trace, the highest frequency is $40 \mathrm{~Hz}$, whereas the low ratio wavelet scalograms skew the power toward
$60 \mathrm{~Hz}$. The higher ratio wavelet, however, resolved each of these individual harmonics at the cost of assigning the power to a specific time. Nevertheless, as the synthetic 
(a) 3 minutes
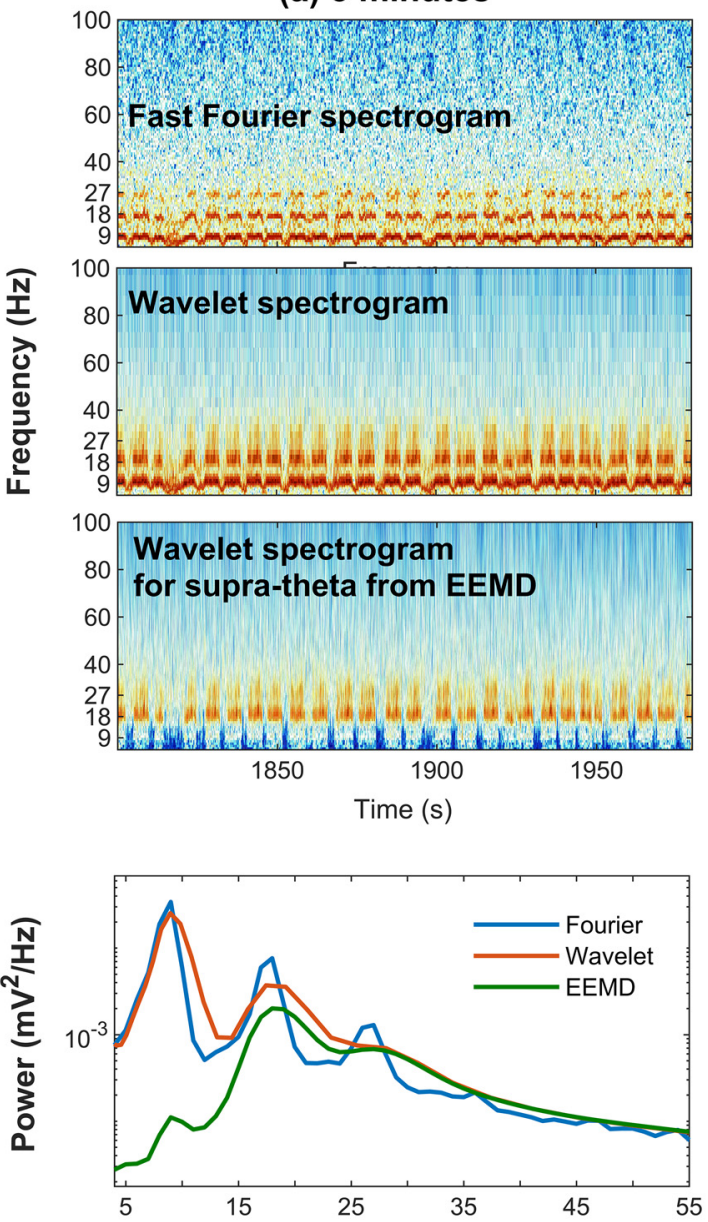

(b) 6 seconds

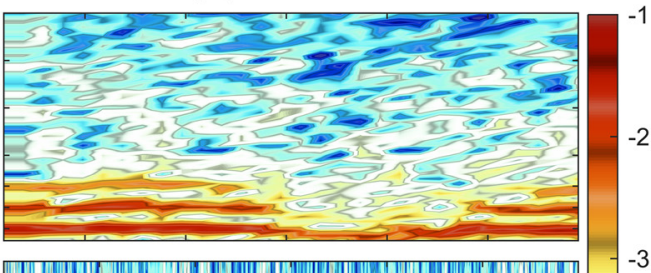

$-3$
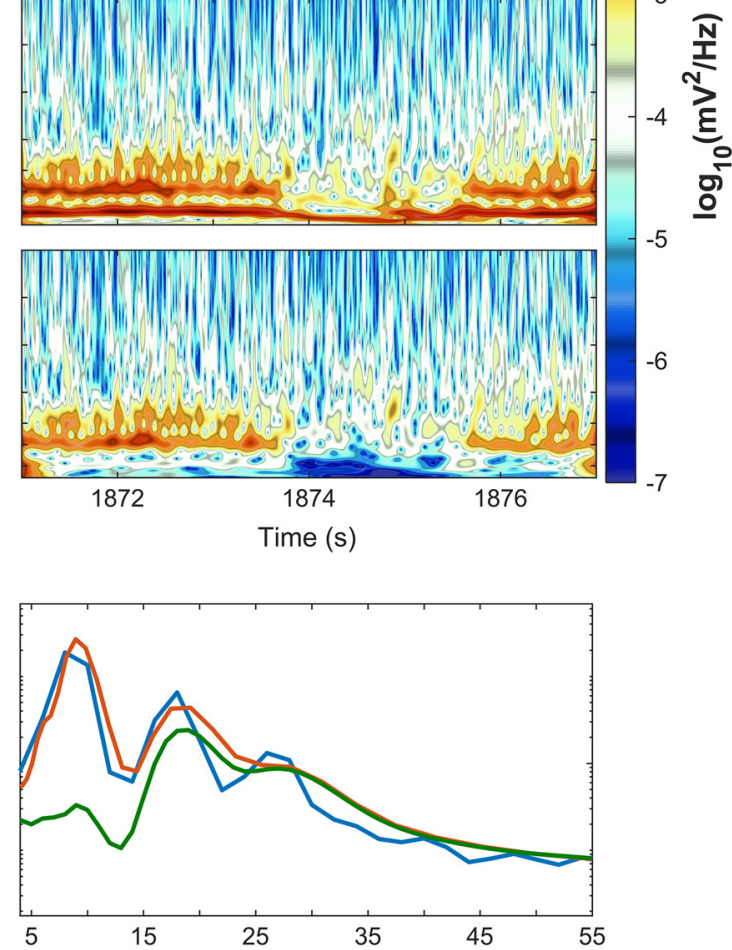

Frequency (Hz)

Figure 12. Spectrograms and power spectra obtained from recordings while rats were running on a maze. Spectrogram and power spectrum were computed for a long run epoch (column $\boldsymbol{a}, 5 \mathrm{~min}$ ) and a short epoch (column $\boldsymbol{b}, 6 \mathrm{~s}$ ). For each epoch, the spectrograms were estimated with three methods: (1) Fourier transform (1st row) with a window length of $1 \mathrm{~s}$ and window increment of $0.1 \mathrm{~s}$; (2) wavelet transform (2nd row) with Morlet wavelet; 3 ) wavelet spectrogram of supra- $\theta$ signal (3rd row). The supra- $\theta$ signal was obtained from EEMD with noise level equaling to 0.5 total variance and ensemble number equaling to 200 . The supra- $\theta$ was defined as the sum of decomposed modes whose central frequencies were larger than $12 \mathrm{~Hz}$. The wavelet spectrogram of supra- $\theta$ was computed with the method described above. Power spectra were computed by averaging the spectrogram over time (bottom row). For both epochs, the Fourier transform identifies $\theta$ and high order harmonics while wavelet analysis tends to resolve a $\theta$ rhythm and a wide-band frequency component $(16-30 \mathrm{~Hz})$. By considering this band as independent from $\theta$ in this manner gives the unintentional representation that there are "bursts" of $\gamma$. Stated differently, the 24- $\mathrm{Hz}$ oscillation is inherently dependent on the nonlinearity of $\theta$ ("sawtooth" shape of $\theta$ ) and thus is incorrectly decomposed by the wavelet.

harmonics are convolved by the lower ratio wavelets $(7$ being the one implemented in the initial description of slow $\gamma$ ) to give the impression of a broad slow $\gamma$ band, LFP collected from the CA1 pyramidal layer is subject to an identical distortion (Fig. 11). When comparing Figure 11 to the initial report of slow $\gamma$ (Colgin et al., 2009, their Fig. 1E) there are dramatic differences in power across frequencies. Although the power spectrum has a relationship such that low frequencies have high power and high frequencies have low power (often described as the $\mathrm{A}=$ $\mathrm{f}^{-\alpha}$ slope), the scalograms in Colgin et al. (2009) suggests that the power in the $>100-\mathrm{Hz}$ band is significantly larger than the $50-\mathrm{Hz}$ band. This is due to the application of a pre-whitening filter resulting in the caveat that "... power magnitudes depicted in these illustrations should be ignored since they do not accurately reflect the actual size of waveforms in the original local field potential recordings" (Colgin et al., 2009, their supplementary information, p. 38). While whitening was implemented to visualize power at higher frequencies, the initial depiction justifying the division of slow $\gamma$ was acknowledged to be an inappropriate representation of the raw data. This issue is compounded when considering the validity of the wavelet parameters selected for decomposition.

As the results of wavelet and EEMD are often portrayed in terms of a time-power-frequency representation, we furthered the comparison using two different time scales of analysis (Fig. 12). Both in the long (3-min Fourier trans- 
form) and short (6s Fourier transform), there is the definite presence of a third-order $\theta$ harmonic $(\sim 24-27 \mathrm{~Hz})$ and a trace of a fourth harmonic $(\sim 32-36 \mathrm{~Hz}$; Fig. 12, top panels). However, as anticipated from the Heisenberg boxes (Fig. 4), the wavelet decomposition as implemented in previous studies results in a convolution and apparent intermittency of the $\sim 24-$ to $27-\mathrm{Hz}$ frequency band that is particularly evident at the 6-s time scale. Because the 24to $27-\mathrm{Hz}$ component is a direct consequence of $\theta$ being skewed and asymmetric (for example, more "saw tooth" shape than a sinusoid; Buzsáki et al., 1983, 1985; Terrazas et al., 2005; Sheremet et al., 2016), this frequency is inherently coupled to the fundamental $8-\mathrm{Hz}$ rhythm (that is, as stationary as $\theta$; not a transient event). The wavelet decomposition as implemented in prior studies, however, artificially detaches the third harmonic from the fundamental by using a different decimation of time and frequency (Fig. 2). This convolution and intermittency offers a strong resemblance to experiments that report different $\theta$ cycles exhibiting unique $\gamma$ signatures (Colgin et al., 2009; Bieri et al., 2014). To an extreme, using EEMD, it has been argued that $\theta$ can mix and match different $\gamma$ frequencies (Bagur and Benchenane, 2018) in support of different memory processes (Lopes-Dos-Santos et al., 2018). Therefore, we also replicated the methods of Lopes-Dos-Santos et al. (2018). Notably, comparing both the long and short temporal epochs of wavelet decomposition of the "supra- $\theta$ " reveals the distorted remnants of the second harmonic of $\theta$ as well as the third harmonic (Fig. 11). Finally, the summary power spectra for each method is presented (Fig. 11, bottom panels) demonstrating the inability of either wavelet, or EEMD followed by wavelet to resolve the $\theta$ harmonics. This perspective gives rise to another issue, that is, although the harmonics of $\theta$ effectively mirror the stationarity of $\theta$, the varying timefrequency boxes of wavelet give the invalid impression of transience.

\section{Slow $\gamma$ and the issue of transience}

The slow $\gamma(25-50 \mathrm{~Hz})$ and "fast $\gamma$ " $(60-100 / 140 \mathrm{~Hz})$ oscillations have often been discussed as being nonstationary and transient (Bieri et al., 2014; Zheng et al., 2015; Lopes-Dos-Santos et al., 2018). Therefore, it is plausible that slow $\gamma$ is so transient as to be averaged out by Fourier decomposition (although leaving one to wonder why frequencies between $50-120 \mathrm{~Hz}$ and ripples are reliably resolved by Fourier decomposition; see Buzsáki et al., 2003 and Johnson and Redish, 2007). While wavelet is capable of detecting the presence of a transient highfrequency event, it is liable to distort the frequency of the same event (as demonstrated in the analyses on the synthetic traces above); power and frequency range quantification will have diminished meaning. To this point, we have demonstrated that wavelet as traditionally implemented is subject to this deformation, but we have not considered the possibility that transient $25-$ to $50-\mathrm{Hz}$ high power bursts exist in the LFP.

Therefore, to cross-validate the detection of slow $\gamma$ events with quantification, we implemented a simple filter method to identify transient events. First, the $25-$ to $55-\mathrm{Hz}$ filtered trace was squared (rectified; Fig. 13). Next, the mean and SD of the envelope of the rectified trace was calculated. As instances in which power (amplitude squared) exceed 2 SDs above the mean has been reported to optimize between detecting slow $\gamma$ events and rejecting noise (Zheng et al., 2015), we mirrored this approach. Thus, although the statistical characteristics of the LFP on the whole change across long time scales, by triggering to a known event, the LFP can be treated as locally stationary, which in turn allows the spectra to be realized (Pesaran et al., 2018). Importantly, this method has also proved sensitive enough to detect the transient low amplitude, high-frequency ripples (Maurer et al., 2006; Skaggs et al., 2007; n.b., the amplitude of ripples are lower than lower frequency events).

Analyzing 2-s epochs centered on high power 25- to $55-\mathrm{Hz}$ events using the multi-taper and previous implemented wavelet methods resulted in two contradictory representations. The Fourier based multi-taper decomposition peaks at a $24 \mathrm{~Hz}$ and potentially a $32-\mathrm{Hz}$ harmonic of $\theta$ which are absent in the wavelet decomposition. In their place is a single, broad band 25- to $55-\mathrm{Hz}$ bump indicative that these events would most likely overlap with the same epochs identified using the method of Bieri et al. (2014). Note that neither of these two decompositions offer a "mixed representation" where both harmonics and slow $\gamma$ are present. Rather, the outcome is dichotomous and dependent on the decomposition. to determine which representation is true to the biology, the high-power epochs were then processed through bicoherence analysis to test for harmonics (Aru et al., 2015). It is worth emphasizing that the wavelet power spectra give the impression that these are indeed high power, $25-$ to $50-\mathrm{Hz}$ events. In the bicoherence plot, however, there is a peak interaction between 17 and $17 \mathrm{~Hz}$ as well as between 8.5 and $25.5 \mathrm{~Hz}$ indicative of $\theta$ harmonics as high as $34 \mathrm{~Hz}$. Therefore, while the method of detecting slow $\gamma$ is "corroborated" by the wavelet decomposition, the high-power $25-$ to $55-\mathrm{Hz}$ events are in fact a biological consequence of higher order $\theta$ harmonics.

To demonstrate that this method is indeed capable of resolving transient events, the same approach was applied to a $120-$ to $250-\mathrm{Hz}$ filtered trace. Despite the intermittent nature of ripples, both wavelet and multi-taper revealed a peak in the ripple band although the wider Heisenberg uncertainty boxes of wavelet distributes the power of ripples over a wider range. Furthermore, the bicoherence analysis reveals interactions of the ripple frequency with a low frequency event. Therefore, in our data, it becomes evident that the spectral decomposition methods selected determine whether slow $\gamma$ or $\theta$ harmonics are observed.

\section{Discussion}

Accuracy describes the proximity of a measurement to the quantity's true value, whereas precision is the degree to which the method yields a consistent result under unchanged circumstances (ISO 5725-1, 1994; VIM, 2004). Evidence in support of slow $\gamma$ has come in the form of the ability of wavelet to reliably achieve the same answer. The 
A

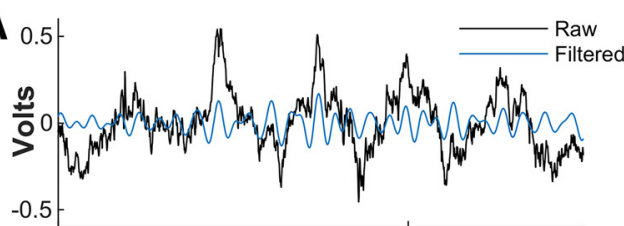

B

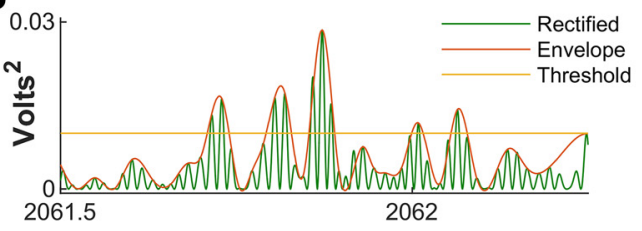

Time (sec)

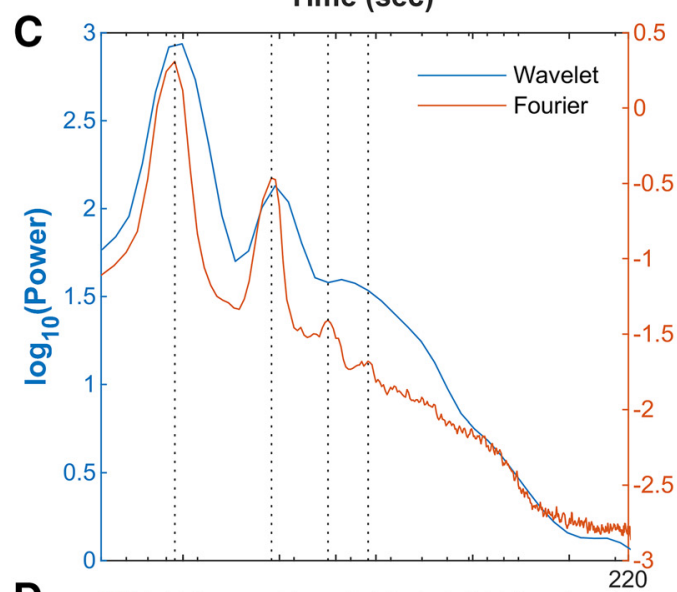

D
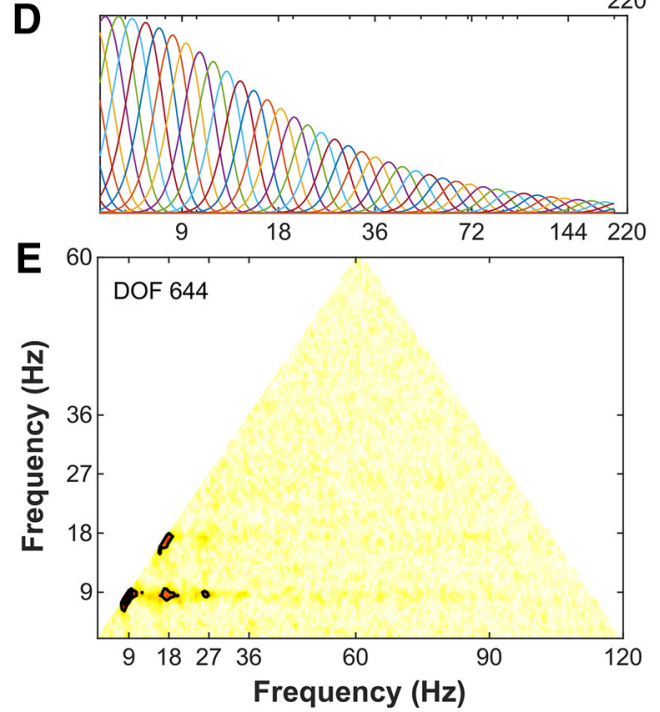

$\mathbf{F}$

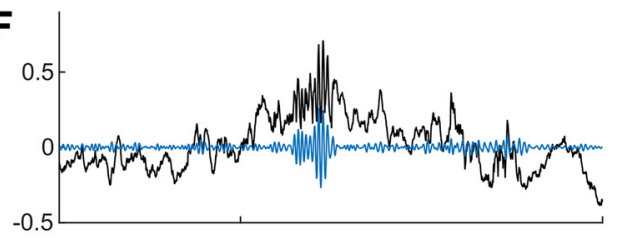

G

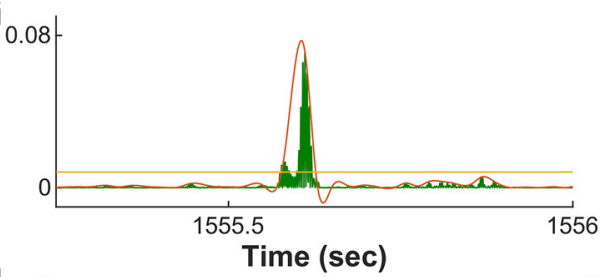

H

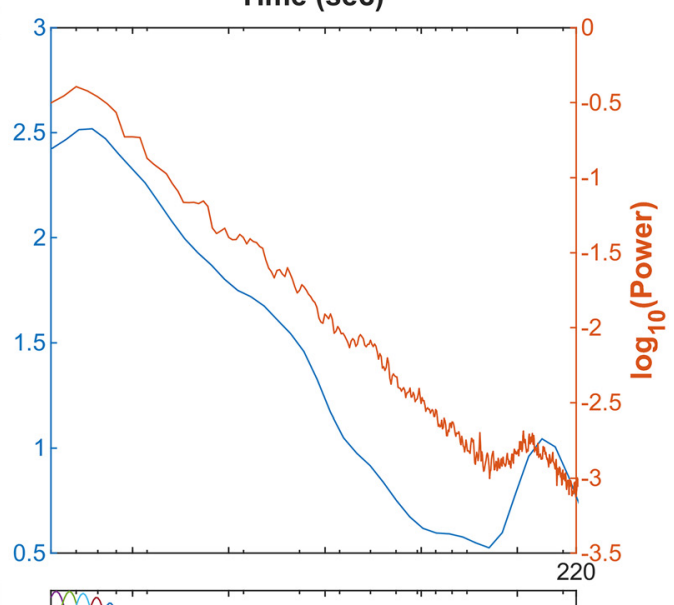

I
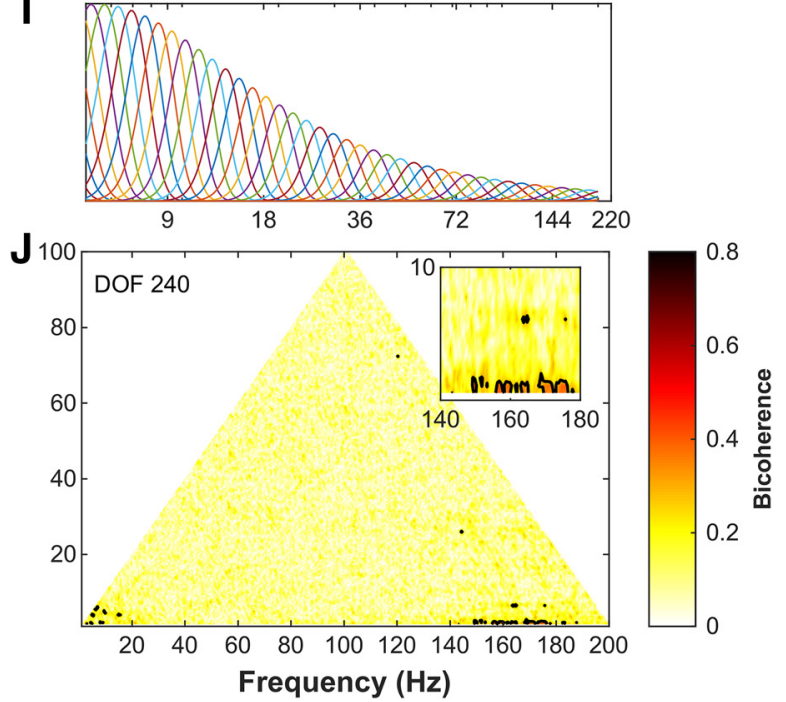

Figure 13. Power based detection of ripples and slow $\gamma$ and the corresponding Fourier and Wavelet decompositions. A, Raw and filtered $(25-50 \mathrm{~Hz})$ trace of pyramidal layer LFP. $\boldsymbol{B}$. High-amplitude $25-$ to $55-\mathrm{Hz}$ events were detected by calculating the mean and SDs of the envelope of the squared filtered trace. $\boldsymbol{C}$, For each instance in which the slow $\gamma$ power exceeded 2 SDs above the mean power, a 2-s window was extracted and processed using either Thomson's multi-taper method or wavelet decomposition using the same approach as Colgin et al. (2009). Interestingly, the wavelet decomposition reveals a slow $\gamma$ bump (suggesting that wavelet would concur that these are slow $\gamma$ epochs), whereas the Fourier decomposition shows multiple bumps indicative of harmonics. $\boldsymbol{D}$, Frequency range of the individual wavelets implemented in Colgin et al. (2009). As the frequency increases so does the range of an individual wavelet which is responsible for the redistribution of power across a broad band. $\boldsymbol{E}$, Bicoherence decomposition of the epochs in which the 25- to 55- $\mathrm{Hz}$ power exceeded 2 SDs above the mean. The significant regions at 18 and $18 \mathrm{~Hz}$ and 9 and $27 \mathrm{~Hz}$ indicate an interaction with the 4th, 36- $\mathrm{Hz}$ harmonic (Sheremet et al., 2016). Therefore, this demonstrates based on the parameters used in prior publications, the wavelet approach is convolving over harmonics of $\theta$ to erroneously give the impression of a slow $\gamma$ bump. $\boldsymbol{F}$, Raw and filtered $(120-250 \mathrm{~Hz})$ trace of pyramidal layer LFP. G, Ripple events were detected when the envelope of the rectified trace exceeded 2 SDs above the mean. $\boldsymbol{H}$, Analyzing each detected instance of a ripple in a 2-s window reveals a significant power in the ripple frequency demonstrating that it is possible to decompose transient events such as high-frequency ripples. $I$, Same 
continued

as $\boldsymbol{D}$. $\boldsymbol{J}$, Bicoherence decomposition of the detected ripple epochs reveals an interaction between low-frequency events at $\sim 2-10 \mathrm{~Hz}$ and a $150-$ to $180-\mathrm{Hz}$ band (see inset). This interaction is most likely a consequence of coupling between ripples and sharp wave related deviations.

present manuscript, however, provides evidence that EEMD and wavelet-based methods, using common parameters, inaccurately represents the LFP. Using data generously provided by the Buzsáki laboratory, we observed cross-frequency coupling between $\theta, \theta$ harmonics, and a unitary 50- to $120-\mathrm{Hz} \gamma$ band (also see Sheremet et al., 2019a). In the power spectra from the CA1 pyramidal layer LFP, there was no detectable peak in the slow $\gamma$ range. These results are largely in agreement with prior publications implementing Fourier decomposition describing $\theta$, harmonics of $\theta$ and a broad, high-frequency unitary $\gamma$ band in CA1 (Buzsáki et al., 2003) and CA3 regions (Johnson and Redish, 2007). The absence of slow $\gamma$ in CA3 is notable as this region is hypothesized to be the communication conduit of slow $\gamma$ to CA1 (Colgin et al., 2009). Fourier does not yield the same values as wavelet.

However, the absence of a peak is not indicative that a fundamental rhythm is absent. A coherence may exist between the LFP and spiking activity of single units (Pesaran et al., 2018). Using data available on the CRCNS.org repository provided by the Buzsáki laboratory, we analyzed the spike frequency of neurons, the spike-LFP coherence, and the depth-of-modulation characteristics. When the firing frequency of spikes were analyzed, there was modulation of both principal cell, and interneuron firing rate by $\theta$ and its first-harmonic at low and high running speeds, but no modulation at the $25-$ to $50-\mathrm{Hz}$ band was observed. These results were buttressed by the spike-LFP coherence analysis, again failing to resolve a slow $\gamma$ interaction. Finally, we analyzed the spike modulation by different oscillatory frequencies within the hippocampus. Notably, there was modulation across all frequencies, although $\theta$ modulation eclipsed all others. As modulation is the default, finding significant modulation with a specific frequency band does not qualify as support for a fundamental rhythm.

In our past studies (Sheremet et al., 2016, 2019a) as well as the present manuscript, we have thrice failed to replicate the initial observation of a $25-$ to $50-\mathrm{Hz}$ oscillation in the CA1 pyramidal layer with either bicoherence analysis or Fourier decomposition (Colgin et al., 2009). If neither spike modulation nor Fourier decomposition provides support for the slow $\gamma$ hypothesis, then what is responsible for the fervent dogma for the phenomenon? Here, we demonstrate that wavelet ratio of 7 reliably decimates a time series into an inaccurate representation.

Fourier decomposition operates by decomposing a time series into sine waves, allowing the construction of a power spectrum by which each frequency is assigned a unitary amplitude value. There is a fixed ratio between time and frequency where increasing frequency resolution comes at the cost of decreasing temporal resolution and vice versa. Wavelet, on the other hand, uses a varying time-frequency decimation in which there is a progressive increase in temporal resolution as frequency increases.
The distortion related to the non-uniform time-frequency decimation was noted early in the application of wavelet to neuroscience data. Tallon-Baudry et al. (1997) investigated the 30- to $70-\mathrm{Hz}$ cortical $\gamma$ rhythm, using the same wavelet ratio of Colgin et al. (2009) of 7, describing the trade-off: "At $20 \mathrm{~Hz}$, this leads to a wavelet duration . . . of $111.4 \mathrm{msec}$ and to a spectral bandwidth . . . of $5.8 \mathrm{~Hz}$, and at $100 \mathrm{~Hz}$ to a duration of $22.2 \mathrm{msec}$ and a bandwidth of 28.6 Hz" (Tallon-Baudry et al., 1997, p. 724). The spectral support for this wavelet generates an overlap between the 21 - to $27-$ and the $28-$ to $36-\mathrm{Hz}$ range such that the power for one $\theta$ harmonic will bleed into the other (Fig. 4). Assuming $125 \mathrm{~ms}$ for a single $\theta$ cycle, the precision of temporal locking measured in this manner will bleed power across $\sim 78-106 \mathrm{~ms}$ for $25-50 \mathrm{~Hz}$, or $>180^{\circ}$. Despite these shortcomings, wavelet was favored for its ability to identify the temporal onset of a transient event (Tiitinen et al., 1993; Sinkkonen et al., 1995). The hippocampal $\gamma$ oscillation could theoretically be considered a transient event necessitating the use of wavelets. A problem arises, however, if the same method used to detect the events is also used to quantify the frequency should it have a trade-off in one dimension (high temporal precision) versus another (low precision in frequency). The non-uniform time-frequency representation distributes the power over a wide frequency band depending on the wavelet parameters used. As demonstrated in Figure 11, the methods using the parameters in the initial description of slow $\gamma$ are incapable of resolving harmonics in a synthetic trace. Rather, power is distributed across a wide band, giving the impression of a non-existent 25- to $50-\mathrm{Hz}$ oscillation. Note that it is possible to construct an appropriate wavelet that can resolve the harmonics (Fig. 11). More recently, the application of EEMD to the LFP in the CA1 pyramidal layer suggests that there are two $\gamma$ bands $<50 \mathrm{~Hz}$ in frequency (Lopes-Dos-Santos et al., 2018). Although it was argued that EEMD could remove harmonics, no verification was presented. When tested against synthetic traces and the LFP, EEMD failed to remove the harmonics (Fig. 10). As the EEMD results were then passed to an aggressive wavelet convolution (Fig. 3) in Lopes-Dos-Santos et al. (2018), the power in the $\theta$ harmonic range was distributed across $25-50 \mathrm{~Hz}$, giving the erroneous impression of low-frequency $\gamma$ bands. These issues extended into the time-frequency spectrograms in which a fourth harmonic was detectable by Fourier, but not resolved with wavelet or EEMD (Fig. 12). Finally, we considered the possibility in which slow $\gamma$ is transient to the point of being averaged out by Fourier decomposition. Epochs were analyzed in which the power of either ripples or slow $\gamma$ exceeded 2 SDs above the average power, allowing potentially non-stationary events to be treated as locally stationary (Pesaran et al., 2018). While Fourier based methods revealed a peak in the ripple frequency in the decomposition, only harmonics were present in the 
epochs in which the 25- to $55-\mathrm{Hz}$ band exceeded 2 SDs above the mean power. The presence of harmonics was verified with bicoherence analysis. Wavelet run on the same epochs, however, convolved over the harmonics and gave the impression of a slow $\gamma$ band.

Moving forward we can offer two suggestions. First, as velocity is known to increase $\theta$ harmonic power (Sheremet et al., 2016, 2019a), future approaches into spectral decomposition should be parameterized by rat-running speed or, at the least, sorted by overall variance (Sheremet et al., 2019b). Second, as analytical toolboxes become more ubiquitous, caution has been emphasized that the user needs to be aware of the caveats or otherwise risk generating mistakes (Marder, 2015). In light of this, we underscore the importance of testing the decomposition method against a synthetic trace with harmonics as well as Fourier decomposition (confirming the validity).

This manuscript questions the validity of analytical approaches used to describe a broadband $25-$ to $50-\mathrm{Hz}$ slow $\gamma$ oscillation in the CA1 pyramidal layer as a fundamental hippocampal oscillation. Here, we demonstrate that different spectral decomposition techniques yield different representations of the data. First, while we can replicate the initial findings of Colgin et al. (2009) and Lopes-Dos-Santos et al. (2018), these decompositions are at odds with the Fourier decomposition. Second, wavelet analysis is unstable to the change of parameters and in fact, drifts toward the Fourier representation when the wavelet is optimized for frequency resolution. Third, Fourier decomposition does not offer a realization that is supportive of the slow $\gamma$ observation, but rather harmonics of $\theta$. Finally, it needs to be re-emphasized that none of these methods offered a mixed representation of slow $\gamma$ superimposed onto the higher order harmonics of $\theta$. In the instance that they coexist, Fourier spectrum would be able to represent both rhythms. However, this is not the case for the present manuscript. The dissociable outcomes of slow $\gamma$ and $\theta$ harmonics were directly related to the decomposition method and parameters selected (as evidenced in the analysis of the synthetic trace). As each decomposition gives a different representation of the underlying time series, they all cannot be high-fidelity representations of the actual biology. Without having access to the data of Colgin et al. (2009) and Lopes-Dos-Santos et al. (2018), we are incapable of determining whether their reports are consistent with Fourier decomposition. Although the absence of slow $\gamma$ in the spectral decomposition is complimented by the lack of neuron action potential modulation in the $25-$ to $50-\mathrm{Hz}$ band, it is necessary to reiterate that the current study does not refute that the brain is capable of exhibiting slow $\gamma$. The detection of such oscillatory events in the studies where wavelet or EEMD analyses were implemented with improperly tuned parameters, however, cannot be used as evidence of the existence of a $25-$ to $50-\mathrm{Hz}$ oscillation that is independent from other frequency bands and meaningful for behavior.

To our knowledge, this is the first attempt to disambiguate slow $\gamma$ from $\theta$ harmonics. If a slow- $\gamma$ rhythm exists, this analysis points, in the least, to the need of disambiguating it from higher order $\theta$ harmonics. After accounting for the harmonics of $\theta$ here, there was little reason to support the slow $\gamma$ hypothesis. Previous work has suggested that the decrease in power in the 25- to $50-\mathrm{Hz}$ range that occurs as a function of velocity is in support of the multiple $\gamma$ hypothesis (Fig. 6). While this figure could be interpreted as support for two dissociable $\gamma$ rhythms, change in $25-$ to $50-\mathrm{Hz}$ power with velocity is, in fact, equivocal. There have been reports of decreasing slow $\gamma$ power with velocity (Kemere et al., 2013), no change in power with velocity (Zheng et al., 2015), or increase in power with velocity (Chen et al., 2011). Bieri and colleagues did not run an explicit analysis, but their highest proportion of slow $\gamma$ events occurred in the middle of the track where the running speed was highest and fewest detected events occurred at the ends of the track where velocity was the lowest (Bieri et al., 2014, their supplemental data), suggesting a positive relationship between power and velocity. As an alternative hypothesis, Ahmed and Mehta (2012) argued that there is a single $\gamma$ frequency which increases with running speed, resulting in a decrease in amplitude of low-frequencies and an increase at higher frequencies. They interpreted their findings as being consistent with the work of Traub et al. (1996), who observed an increase in $\gamma$ frequency as a function of feedforward drive. To our knowledge, this is the first description of the hippocampal power spectra undergoing a redistribution of power across frequencies as a function of afferent input.

In accord with this, we observe a spectral reorganization in which some bands increase in power while others decrease as running speed increases (Sheremet et al., 2019a, b). The drops in power occur between 1 and 6,10 and 14, 19 and 23, and 25 and $40 \mathrm{~Hz}$ (Figs. 5, 6). This type of spectral reorganization has been described before and has a long history. Norbert Wiener observed an increase in the $10-\mathrm{Hz} \alpha$ power that was associated with dips (loss of power) in the adjacent frequencies (Wiener, 1965, 1966). The insight was that the global oscillation in the EEG arose from the collective action of the population. As described by Steven Strogatz in his computational model of the phenomenon is: ". . . that the oscillators interact by pulling on each other's frequencies - if an oscillator is ahead of the group, the group tends to slow it down. If it is going too slowly, the group tends to speed it up. In this way the population of oscillators can achieve a collective enhancement of precision." (Strogatz, 1994, p. 122). Oscillations of the hippocampus do not reside within a single cell or a pair of cells but across the entire population. As the hippocampus becomes more excitable with running speed, the oscillatory dynamics achieve a "collective enhancement of precision" (for a similar perspective, see Churchland et al., 2010). Therefore, the power in 25- to $50-\mathrm{Hz}$ frequency band at low running speed is a consequence of incohesive oscillatory dynamics among the neurons when not entrained (making the claim that slow $\gamma$ is a fundamental rhythm dubious; oscillations tend to be equated to entrainment, not incoherence). Once input is large enough, the oscillators fall into an en masse alignment, enhancing the power in some frequency bands at the expense of others. 
Of course, the question arises: What empirical data would be needed to support an assertion of slow $\gamma$ ?. The consideration of this question raises the core issue: what is a rhythm? Traditionally, a "rhythm" has been identified visually, as peaks above some background level. For instance, in the construction of the PSD, $\theta$ exhibits a clear peak above the $1 / f^{\alpha}$ slope. Identifying peaks at higher frequencies are more difficult and a clear deviation from $1 / f^{\alpha}$ for a broad $50-$ to $120-\mathrm{Hz} \gamma$ often only becomes evident at high running speeds (Fig. 6) when neuron firing rate is elevated, and there is more energy in the hippocampal network (Sheremet et al., 2016, 2019a). The implied assumption is that the peaks above noise (that is, the rhythms) are the "meaningful" signal and the background is "meaningless," that is, noise. While the signal/ noise dichotomy is ubiquitous in physics, it serves the purpose of defining the scale of interest, by acknowledging that the scale and physics governing the signal are fundamentally different from that of the noise. Fourier and wavelet decompositions both yield representations in which there is power across all frequencies (Fig. 9). Thus, if decomposition reveals power across all frequencies, is it meaningful to segregate any frequency band and ascribe it meaning without understanding how it is different from any of the other frequencies in terms of the network dynamics and biophysical mechanisms that underlie that frequency? From an alternative perspective, consider that a single dynamic process in the network is capable of casting multiple frequencies (akin to an EKG). In this sense, using a correlative approach to divvy up frequencies relative to behavior is not likely to provide much in the way of insight with respect to how the brain organizes behavior (Buzsáki, 2005). In fact, the notion of a multiplexed "spectral fingerprint" (Canolty et al., 2010; Siegel et al., 2012; Knight and Eichenbaum, 2013; Watrous et al., 2013; Akam and Kullmann, 2014; McLelland and VanRullen, 2016) in which different frequency bands independently support unique aspects of cognition should be rigorously tested alongside the alternative model of a spectral energy cascade (Sheremet et al., 2019b).

It should be noted here that, from the biological perspective, the concept of multiplexing is physiologically untenable as it is inaccurate to assume that the LFP recorded near the soma of CA1 pyramidal cells is a linear mirror of the LFP from its afferent inputs. The relationship between the LFP and the source is not this simple or straightforward (Herreras, 2016). With respect to the "routing hypothesis" (Colgin et al., 2009), it needs to be understood that CA3 inputs terminate into the stratum radiatum (more proximal to the soma) whereas entorhinal synapses terminate on the distal dendrites in the lacunosum-moleculare (Amaral and Witter, 1989; Witter and Amaral, 2004), further from the CA1 stratum pyramidale. However, dendrites operate as low pass filters (Golding et al., 2005) in which synaptic input may be $\gamma$ paced at the dendrites, but become a bolus of lowfrequency $(\theta)$ input at the soma (Vaidya and Johnston, 2013). Rather than routing information, the Schaffer collateral and perforant pathway inputs can be parsimoniously described as providing energy into CA1. These synaptic inputs are going to interact, as any depolarization or hyperpolarization of the cell is going to affect the ionic driving force. In other words, Schaffer collateral input will influence entorhinal cortical input and vice versa. Thus, $\gamma$ in the CA1 pyramidal layer is not a mirror of its afferent input, but a consequence of local interactions between pyramidal neurons and interneurons (Marshall et al., 2002; Maurer et al., 2006). Should excitatory drive into CA1 come in bouts of $\theta$, then increasing the power of the $\theta$-paced afferent input promotes more local $\gamma$ rhythmicity (describing an energy cascade).

The spectral energy cascade hypothesis (Bak et al., 1987; Buzsáki, 2006; Sheremet et al., 2019b) proposes that there is a nonlinear interaction across scales, resulting in both energy exchange and phase coupling between different scales (frequencies). With respect to hippocampal LFP, the amplitude and phase of $\gamma$ will be inherently coupled to the amplitude and phase of $\theta$. The amplitude and phase of all meaningful high-frequency rhythms will be coupled to higher power/lower frequency rhythms. Should this be true, there is corollary evidence that the $\theta$ rhythm provides the necessary energetic drive for neurons to engage in $\gamma$ rhythmicity. A big peak in $\theta$ means "big energy" to lower amplitude, higher frequency rhythms, including $\gamma$. Continuing this line of logic, as noted above, peak and phase are inherently coupled such that the highest amplitude is defined as phase zero. From the energy cascade perspective, where $\theta$ forces $\gamma$, why is it surprising that the phase of $\theta$ exhibits a relationship to the power of $\gamma$ ? One would suppose that external, $\theta$-paced input from the entorhinal cortex and septum would trigger a cascade of higher frequency events in the hippocampus and stronger input results in a larger amplitude $\gamma$. Thus, the rhythms of the hippocampus should not be considered to have orthogonal relationships with each other. Instead, the most straightforward description is that all rhythms in the LFP are interdependent, coupled by the cascade of energy across spatial and temporal (frequency) scales.

Taken together, the data presented here provide a parsimonious explanation to why wavelet and EEMD analysis results in the appearance of a slow $\gamma$ band. For both decomposition methods, this involved the parameters that are used to optimize temporal resolution at the cost of frequency resolution. Admittedly, this study cannot claim to have done an exhaustive investigation of every brain region, the entire parameter space, and all possible decomposition methods. Thus, it is our intention that this work will serve as the impetus for future research that includes additional decomposition tools and brain regions.

\section{References}

Abry P, Gonçalves P, Flandrin P (1993) Wavelet-based spectral analysis of $1 / f$ processes. In: Acoustics, speech, and signal processing, pp 237-240. Piscataway, NJ: IEEE Service Center.

Ahmed OJ, Mehta MR (2012) Running speed alters the frequency of hippocampal gamma oscillations. J Neurosci 32:7373-7383.

Amaral DG, Witter MP (1989) The three-dimensional organization of the hippocampal formation: a review of anatomical data. Neuroscience 31:571-591. 
Akam T, Kullmann DM (2014) Oscillatory multiplexing of population codes for selective communication in the mammalian brain. Nat Rev Neurosci 15:111-122.

Aru J, Priesemann V, Wibral M, Lana L, Pipa G, Singer W, Vicente R (2015) Untangling cross-frequency coupling in neuroscience. Curr Opin Neurobiol 31:51-61.

Bagur S, Benchenane K (2018) The theta rhythm mixes and matches gamma oscillations cycle by cycle. Neuron 100:768-771.

Bak P, Tang C, Wiesenfeld K (1987) Self-organized criticality: an explanation of the 1/f noise. Phys Rev Lett 59:381-384.

Barnett TP, Johnson LC, Naitoh P, Hicks N, Nute C (1971) Bispectrum analysis of electroencephalogram signals during waking and sleeping. Science 172:401-402.

Belluscio MA, Mizuseki K, Schmidt R, Kempter R, Buzsáki G (2012) Cross-frequency phase-phase coupling between $\theta$ and $\gamma$ oscillations in the hippocampus. J Neurosci 32:423-435.

Berényi A, Somogyvári Z, Nagy AJ, Roux L, Long JD, Fujisawa S, Stark E, Leonardo A, Harris TD, Buzsáki G (2014) Large-scale, high-density (up to 512 channels) recording of local circuits in behaving animals. J Neurophysiol 111:1132-1149.

Bieri KW, Bobbitt KN, Colgin LL (2014) Slow and fast $\gamma$ rhythms coordinate different spatial coding modes in hippocampal place cells. Neuron 82:670-681.

Bragin A, Jandó G, Nádasdy Z, Hetke J, Wise K, Buzsáki G (1995) Gamma $(40-100 \mathrm{~Hz})$ oscillation in the hippocampus of the behaving rat. J Neurosci 15:47-60.

Breen TE, Morzorati SL (1996) Innate differences in medial septal area burst firing neurons and the hippocampal theta rhythm during ambulation in selectively bred rat lines. Brain Res 714:156-164.

Briggs WL (1995) The DFT: an owners' manual for the discrete Fourier transform. Philadelphia: SIAM.

Buhl DL, Harris KD, Hormuzdi SG, Monyer H, Buzsáki G (2003) Selective impairment of hippocampal gamma oscillations in connexin-36 knock-out mouse in vivo. J Neurosci 23:1013-1018.

Bullock TH, Buzsáki G, McClune MC (1990) Coherence of compound field potentials reveals discontinuities in the CA1-subiculum of the hippocampus in freely-moving rats. Neuroscience 38:609-619.

Bullock TH, Achimowicz JZ, Duckrow RB, Spencer SS, Iragui-Madoz VJ (1997) Bicoherence of intracranial EEG in sleep, wakefulness and seizures. Electroencephalogr Clin Neurophysiol 103:661-678.

Buzsáki G (2005) Theta rhythm of navigation: link between path integration and landmark navigation, episodic and semantic memory. Hippocampus 15:827-840.

Buzsáki G (2006) Rhythms of the brain. New York: Oxford University Press.

Buzsáki G, Draguhn A (2004) Neuronal oscillations in cortical networks. Science 304:1926-1929.

Buzsáki G, Wang XJ (2012) Mechanisms of gamma oscillations. Annu Rev Neurosci 35:203-225.

Buzsáki G, Schomburg EW (2015) What does gamma coherence tell us about inter-regional neural communication? Nat Neurosci 18: 484-489.

Buzsáki G, Leung LW, Vanderwolf CH (1983) Cellular bases of hippocampal EEG in the behaving rat. Brain Res 287:139-171.

Buzsáki G, Rappelsberger P, Kellényi L (1985) Depth profiles of hippocampal rhythmic slow activity ('theta rhythm') depend on behaviour. Electroencephalogr Clin Neurophysiol 61:77-88.

Buzsáki G, Czopf J, Kondákor I, Kellényi L (1986) Laminar distribution of hippocampal rhythmic slow activity (RSA) in the behaving rat: current-source density analysis, effects of urethane and atropine. Brain Res 365:125-137.

Buzsáki G, Buhl DL, Harris KD, Csicsvari J, Czéh B, Morozov A (2003) Hippocampal network patterns of activity in the mouse. Neuroscience 116:201-211.

Buzsáki G, Anastassiou CA, Koch C (2012) The origin of extracellular fields and currents-EEG, ECoG, LFP and spikes. Nat Rev Neurosci 13:407-420.

Cabral HO, Vinck M, Fouquet C, Pennartz CM, Rondi-Reig L, Battaglia FP (2014) Oscillatory dynamics and place field maps reflect hippocampal ensemble processing of sequence and place memory under NMDA receptor control. Neuron 81:402-415.

Cappaert NL, Lopes da Silva FH, Wadman WJ (2009) Spatiotemporal dynamics of theta oscillations in hippocampal-entorhinal slices. Hippocampus 19:1065-1077.

Canolty RT, Ganguly K, Kennerley SW, Cadieu CF, Koepsell K, Wallis JD, Carmena JM (2010) Oscillatory phase coupling coordinates anatomically dispersed functional cell assemblies. Proc Natl Acad Sci U S A 107:17356-17361.

Carr MF, Karlsson MP, Frank LM (2012) Transient slow gamma synchrony underlies hippocampal memory replay. Neuron 75: 700-713.

Chen Z, Resnik E, McFarland JM, Sakmann B, Mehta MR (2011) Speed controls the amplitude and timing of the hippocampal gamma rhythm. PLoS One 6:e21408.

Chrobak JJ, Buzsáki G (1998) Gamma oscillations in the entorhinal cortex of the freely behaving rat. J Neurosci 18:388-398.

Churchland MM, Yu BM, Cunningham JP, Sugrue LP, Cohen MR, Corrado GS, Newsome WT, Clark AM, Hosseini P, Scott BB, Bradley DC, Smith MA, Kohn A, Movshon JA, Armstrong KM, Moore T, Chang SW, Snyder LH, Lisberger SG, Priebe NJ, et al. (2010) Stimulus onset quenches neural variability: a widespread cortical phenomenon. Nat Neurosci 13:369-378.

Coenen AM (1975) Frequency analysis of rat hippocampal electrical activity. Physiol Behav 14:391-394.

Colgin LL (2015a) Theta-gamma coupling in the entorhinalhippocampal system. Curr Opin Neurobiol 31:45-50.

Colgin LL (2015b) Do slow and fast gamma rhythms correspond to distinct functional states in the hippocampal network? Brain Res 1621:309-315

Colgin LL, Denninger T, Fyhn M, Hafting T, Bonnevie T, Jensen O, Moser MB, Moser El (2009) Frequency of gamma oscillations routes flow of information in the hippocampus. Nature 462:353357.

Coppi B, Rosenbluth M, Sudan R (1969) Nonlinear interactions of positive and negative energy modes in rarefied plasmas (I). Ann Phys 55:207-247.

Cowen SL, Gray DT, Wiegand JL, Schimanski LA, Barnes CA (2018) Age-associated changes in waking hippocampal sharp-wave ripples. Hippocampus. Advance online publication. Retrieved July 7 , 2018. doi: 10.1002/hipo.23005.

Csicsvari J, Jamieson B, Wise KD, Buzsáki G (2003) Mechanisms of gamma oscillations in the hippocampus of the behaving rat. Neuron 37:311-322.

Czurkó A, Hirase H, Csicsvari J, Buzsáki G (1999) Sustained activation of hippocampal pyramidal cells by 'space clamping' in a running wheel. Eur J Neurosci 11:344-352.

Daubechies I (1988) Orthonormal bases of compactly supported wavelets. Commun Pure Appl Math 41:909-996.

Daubechies I (1992) Ten lectures on wavelets. Philadelphia: SIAM.

Dumermuth G, Huber PJ, Kleiner B, Gasser T (1971) Analysis of the interrelations between frequency bands of the EEG by means of the bispectrum. A preliminary study. Electroencephalogr Clin Neurophysiol 31:137-148.

Dvorak D, Radwan B, Sparks FT, Talbot ZN, Fenton AA (2018) Control of recollection by slow gamma dominating mid-frequency gamma in hippocampus CA1. PLoS Biol 16:e2003354.

Elgar S (1987) Relationships involving third moments and bispectra of a harmonic process. IEEE Acous Speech Signal Proc 35:17251726.

Fernández-Ruiz A, Oliva A, Nagy GA, Maurer AP, Berényi A, Buzsáki G (2017) Entorhinal-CA3 dual-input control of spike timing in the hippocampus by theta-gamma coupling. Neuron 93:12131226.e5.

Gabor D (1946) Theory of communication. Part 1: the analysis of information. J Inst Elec Eng 93:429-441.

Gledhill RJ (2003) Methods for investigating conformational change in biomolecular simulations. A dissertation for the degree of Doctor of Philosophy at the Department of Chemistry, the University of Southampton, Southampton. 
Gloveli T, Dugladze T, Rotstein HG, Traub RD, Monyer H, Heinemann $\mathrm{U}$, Whittington MA, Kopell NJ (2005) Orthogonal arrangement of rhythm-generating microcircuits in the hippocampus. Proc Natl Acad Sci USA 102:13295-13300.

Golding NL, Mickus TJ, Katz Y, Kath WL, Spruston N (2005) Factors mediating powerful voltage attenuation along CA1 pyramidal neuron dendrites. J Physiol 568:69-82.

Goupillaud P, Grossmann A, Morlet J (1984) Cycle-octave and related transforms in seismic signal analysis. Geoexploration 23:85102.

Grossmann A, Morlet J (1984) Decomposition of Hardy functions into square integrable wavelets of constant shape. SIAM J Math Analysis 15:723-736.

Hagihira S, Takashina M, Mori T, Mashimo T, Yoshiya I (2001) Practical issues in bispectral analysis of electroencephalographic signals. Anesth Analg 93:966-970.

Harper RM (1971) Frequency changes in hippocampal electrical activity during movement and tonic immobility. Physiol Behav 7:55-58.

Harris B (1967) Spectral analysis of time series. New York: Wiley.

Hasselmann K, Munk W, MacDonald G (1963) Bispectrum of ocean waves. In: Time series analysis (Rosenblatt M, ed), pp 125-139. New York: Wiley.

Haubrich RA, MacKenzie GS (1965) Earth noise, 5 to 500 millicycles per second: 2. Reaction of the Earth to oceans and atmosphere. J Geophys Res 70:1429-1440.

Herreras O (2016) Local field potentials: myths and misunderstandings. Front Neural Circuits 10:101.

Insel N, Patron LA, Hoang LT, Nematollahi S, Schimanski LA, Lipa P, Barnes CA (2012) Reduced gamma frequency in the medial frontal cortex of aged rats during behavior and rest: implications for age-related behavioral slowing. J Neurosci 32:16331-16344.

ISO 5725-1 (1994) Accuracy (trueness and precision) of measurement methods and results-part 1: general principles and definitions. Geneva: International Organization for Standardization.

Jackson J, Goutagny R, Williams S (2011) Fast and slow $\gamma$ rhythms are intrinsically and independently generated in the subiculum. J Neurosci 31:12104-12117.

Jacobson TK, Howe MD, Schmidt B, Hinman JR, Escabí MA, Markus EJ (2013) Hippocampal theta, gamma, and theta-gamma coupling: effects of aging, environmental change, and cholinergic activation. J Neurophysiol 109:1852-1865.

Johnson A, Redish AD (2007) Neural ensembles in CA3 transiently encode paths forward of the animal at a decision point. $J$ Neurosci 27:12176-12189.

Kemere C, Carr MF, Karlsson MP, Frank LM (2013) Rapid and continuous modulation of hippocampal network state during exploration of new places. PLoS One 8:e73114.

Kim YC, Powers EJ (1979) Digital bispectral analysis and its applications to nonlinear wave interactions. IEEE Trans Plasma Sci 7:120-131.

Knight RT, Eichenbaum H (2013) Multiplexed memories: a view from human cortex. Nat Neurosci 16:257-258.

Kovach CK, Oya H, Kawasaki H (2018) The bispectrum and its relationship to phase-amplitude coupling. Neuroimage 173:518539.

Lansink CS, Meijer GT, Lankelma JV, Vinck MA, Jackson JC, Pennartz CM (2016) Reward expectancy strengthens CA1 theta and beta band synchronization and hippocampal-ventral striatal coupling. J Neurosci 36:10598-10610.

Lasztóczi B, Klausberger T (2014) Layer-specific GABAergic control of distinct gamma oscillations in the CA1 hippocampus. Neuron 81:1126-1139.

Lasztóczi B, Klausberger T (2016) Hippocampal place cells couple to three different gamma oscillations during place field traversal. Neuron 91:34-40.

Lee MG, Chrobak JJ, Sik A, Wiley RG, Buzsáki G (1994) Hippocampal theta activity following selective lesion of the septal cholinergic system. Neuroscience 62:1033-1047.
Leung LS (1982) Nonlinear feedback model of neuronal populations in hippocampal CAl region. J Neurophysiol 47:845-868.

Leung LS (1984) Theta rhythm during REM sleep and waking: correlations between power, phase and frequency. Electroencephalogr Clin Neurophysiol 58:553-564.

Leung LS, Vanderwolf CH (1980) Behavior-dependent evoked potentials in the hippocampal CA1 region of the rat. II. Effect of eserine, atropine, ether and pentobarbital. Brain Res 198:119-133.

Leung LW, Lopes da Silva FH, Wadman WJ (1982) Spectral characteristics of the hippocampal EEG in the freely moving rat. Electroencephalogr Clin Neurophysiol 54:203-219.

Leung LS, Canning KJ, Shen B (2005) Hippocampal afterdischarges after $\mathrm{GABA}(\mathrm{B})$-receptor blockade in the freely moving rat. Epilepsia 46:203-216.

Leung LW (1985) Spectral analysis of hippocampal EEG in the freely moving rat: effects of centrally active drugs and relations to evoked potentials. Electroencephalogr Clin Neurophysiol 60:6577.

Leung LW, Buzsáki G (1983) Spectral analysis of hippocampal unit train in relation to hippocampal EEG. Electroencephalogr Clin Neurophysiol 56:668-671.

Li X, Li D, Voss LJ, Sleigh JW (2009) The comodulation measure of neuronal oscillations with general harmonic wavelet bicoherence and application to sleep analysis. Neuroimage 48:501-514.

Lighthill MJ (1958) An introduction to Fourier analysis and generalised functions. Cambridge: Cambridge University Press.

Lopes-Dos-Santos V, van de Ven GM, Morley A, Trouche S, CampoUrriza N, Dupret D (2018) Parsing hippocampal theta oscillations by nested spectral components during spatial exploration and memory-guided behavior. Neuron 100:940-952.e7.

Mallat S (1999) A wavelet tour of signal processing. Amsterdam: Elsevier.

Marder E (2015) Understanding brains: details, intuition, and big data. PLoS Biol 13:e1002147.

Marshall L, Henze DA, Hirase H, Leinekugel X, Dragoi G, Buzsáki G (2002) Hippocampal pyramidal cell-interneuron spike transmission is frequency dependent and responsible for place modulation of interneuron discharge. J Neurosci 22:RC197.

Masimore B, Kakalios J, Redish AD (2004) Measuring fundamental frequencies in local field potentials. J Neurosci Methods 138:97105.

Masimore B, Schmitzer-Torbert NC, Kakalios J, Redish AD (2005) Transient striatal gamma local field potentials signal movement initiation in rats. Neuroreport 16:2021-2024.

Masuda A, Kuo YY (1981) A note on the imaginary part of bispectra. Deep Sea Res 28:213-222.

Maurer AP, Vanrhoads SR, Sutherland GR, Lipa P, McNaughton BL (2005) Self-motion and the origin of differential spatial scaling along the septo-temporal axis of the hippocampus. Hippocampus 15:841-852.

Maurer AP, Cowen SL, Burke SN, Barnes CA, McNaughton BL (2006) Phase precession in hippocampal interneurons showing strong functional coupling to individual pyramidal cells. J Neurosci 26:13485-13492.

McLelland D, VanRullen R (2016) Theta-Gamma Coding Meets Communication-through-Coherence: Neuronal Oscillatory Multiplexing Theories Reconciled. PLoS Comput Biol 12:e1005162.

McNaughton BL, Barnes CA, O'Keefe J (1983) The contributions of position, direction, and velocity to single unit activity in the hippocampus of freely-moving rats. Exp Brain Res 52:41-49.

Mitzdorf U (1985) Current source-density method and application in cat cerebral cortex: investigation of evoked potentials and EEG phenomena. Physiol Rev 65:37-100.

Mizuseki K, Buzsáki G (2013) Preconfigured, skewed distribution of firing rates in the hippocampus and entorhinal cortex. Cell Rep 4:1010-1021.

Mizuseki K, Diba K, Pastalkova E, Teeters J, Sirota A, Buzsáki G (2014) Neurosharing: large-scale data sets (spike, LFP) recorded from the hippocampal-entorhinal system in behaving rats. F1000Res 3:98. 
Montgomery SM, Buzsáki G (2007) Gamma oscillations dynamically couple hippocampal $\mathrm{CA} 3$ and $\mathrm{CA} 1$ regions during memory task performance. Proc Natl Acad Sci USA 104:14495-14500.

Montgomery SM, Sirota A, Buzsáki G (2008) Theta and gamma coordination of hippocampal networks during waking and rapid eye movement sleep. J Neurosci 28:6731-6741.

Morris R, Hagan J (1983) Hippocampal electrical activity and ballistic movement. In: Neurobiology of the hippocampus, pp 321-331. London: Academic Press.

Muthuswamy J, Sherman DL, Thakor NV (1999) Higher-order spectral analysis of burst patterns in EEG. IEEE Trans Biomed Eng 46:92-99.

Ning TK, Bronzino JD (1989) Bispectral analysis of the rat EEG during various vigilance states. IEEE Trans Biomed Eng 36:497-499.

Ning T, Bronzino JD (1993) Nonlinear analysis of the hippocampal subfields of CA1 and the dentate gyrus. IEEE Trans Biomed Eng 40:870-876.

Pastalkova E, Wang Y, Mizuseki K, Buzsáki G (2015) Simultaneous extracellular recordings from left and right hippocampal areas CA1 and right entorhinal cortex from a rat performing a left/right alternation task and other behaviors. Available at https://doi.org/ 10.6080/KOKS6PHF.

Penttonen M, Kamondi A, Acsády L, Buzsáki G (1998) Gamma frequency oscillation in the hippocampus of the rat: intracellular analysis in vivo. Eur J Neurosci 10:718-728.

Percival DB, Walden AT (1993) Spectral analysis for physical applications. Cambridge: Cambridge University Press.

Pesaran B, Vinck M, Einevoll GT, Sirota A, Fries P, Siegel M, Truccolo W, Schroeder CE, Srinivasan R (2018) Investigating large-scale brain dynamics using field potential recordings: analysis and interpretation. Nat Neurosci 21:903-919.

Petsche H, Stumpf C (1960) Topographic and toposcopic study of origin and spread of the regular synchronized arousal pattern in the rabbit. Electroencephalogr Clin Neurophysiol 12:589-600.

Pradhan C, Jena SK, Nadar SR, Pradhan N (2012) Higher-order spectrum in understanding nonlinearity in EEG rhythms. Comput Math Methods Med 2012:206857.

Priestley MB (1981) Spectral analysis and time series. London: Academic Press.

Quilichini P, Sirota A, Buzsáki G (2010) Intrinsic circuit organization and theta-gamma oscillation dynamics in the entorhinal cortex of the rat. J Neurosci 30:11128-11142.

Rangel LM, Chiba AA, Quinn LK (2015) Theta and beta oscillatory dynamics in the dentate gyrus reveal a shift in network processing state during cue encounters. Front Syst Neurosci 9:96.

Rangel LM, Rueckemann JW, Riviere PD, Keefe KR, Porter BS, Heimbuch IS, Budlong CH, Eichenbaum H (2016) Rhythmic coordination of hippocampal neurons during associative memory processing. Elife 5:e09849.

Rappelsberger P, Pockberger H, Petsche H (1981) Current source density analysis: methods and application to simultaneously recorded field potentials of the rabbit's visual cortex. Pflugers Arch 389:159-170.

Rezvova IR, Frolov AA, Markevich VA (1995) An investigation of hippocampal theta rhythm of rats as a nonlinear dynamic process. Neurosci Behav Physiol 25:419-426.

Rivas J, Gaztelu JM, García-Austt E (1996) Changes in hippocampal cell discharge patterns and theta rhythm spectral properties as a function of walking velocity in the guinea pig. Exp Brain Res 108:113-118.

Roads C (2004) Microsound. Cambridge, MA: The MIT Press.

Robbe D, Montgomery SM, Thome A, Rueda-Orozco PE, McNaughton BL, Buzsáki G (2006) Cannabinoids reveal importance of spike timing coordination in hippocampal function. Nat Neurosci 9:1526-1533.

Rosenblatt M, Van Ness JW (1965) Estimation of the bispectrum. Ann Math Statist 36:1120-1136.

Sabolek HR, Penley SC, Hinman JR, Bunce JG, Markus EJ, Escabi M, Chrobak JJ (2009) Theta and gamma coherence along the septotemporal axis of the hippocampus. J Neurophysiol 101: 1192-1200.

Scheffer-Teixeira R, Tort AB (2016) On cross-frequency phasephase coupling between theta and gamma oscillations in the hippocampus. Elife 5:e20515.

Scheffer-Teixeira R, Belchior H, Caixeta FV, Souza BC, Ribeiro S, Tort AB (2012) Theta phase modulates multiple layer-specific oscillations in the CA1 region. Cereb Cortex 22:2404-2414.

Schomburg EW, Fernández-Ruiz A, Mizuseki K, Berényi A, Anastassiou CA, Koch C, Buzsáki G (2014) Theta phase segregation of input-specific gamma patterns in entorhinal-hippocampal networks. Neuron 84:470-485.

Schwartz L (1950) Theorie des distributions. Paris: Hermann.

Senior TJ, Huxter JR, Allen K, O’Neill J, Csicsvari J (2008) Gamma oscillatory firing reveals distinct populations of pyramidal cells in the CA1 region of the hippocampus. J Neurosci 28:2274-2286.

Shahbazi Avarvand F, Bartz S, Andreou C, Samek W, Leicht G, Mulert C, Engel AK, Nolte G (2018) Localizing bicoherence from EEG and MEG. Neuroimage 174:352-363.

Shen J, Barnes CA, McNaughton BL, Skaggs WE, Weaver KL (1997) The effect of aging on experience-dependent plasticity of hippocampal place cells. J Neurosci 17:6769-6782.

Sheremet A, Burke SN, Maurer AP (2016) Movement enhances the nonlinearity of hippocampal theta. J Neurosci 36:4218-4230.

Sheremet A, Kennedy JP, Qin Y, Zhou Y, Lovett SD, Burke SN, Maurer AP (2019a) Theta-gamma cascades and running speed. J Neurophysiol 121:444-458.

Sheremet A, Qin Y, Kennedy JP, Zhou Y, Maurer AP (2019b) Wave turbulence and energy cascade in the hippocampus. Front Syst Neurosci 12:62.

Siegel M, Donner TH, Engel AK (2012) Spectral fingerprints of largescale neuronal interactions. Nat Rev Neurosci 13:121-134.

Sigl JC, Chamoun NG (1994) An introduction to bispectral analysis for the electroencephalogram. J Clin Monit 10:392-404.

Sinkkonen J, Tiitinen H, Näätänen R (1995) Gabor filters: an informative way for analysing event-related brain activity. J Neurosci Methods 56:99-104.

Sirota A, Montgomery S, Fujisawa S, Isomura Y, Zugaro M, Buzsáki $G$ (2008) Entrainment of neocortical neurons and gamma oscillations by the hippocampal theta rhythm. Neuron 60:683-697.

Skaggs WE, McNaughton BL, Wilson MA, Barnes CA (1996) Theta phase precession in hippocampal neuronal populations and the compression of temporal sequences. Hippocampus 6:149-172.

Skaggs WE, McNaughton BL, Permenter M, Archibeque M, Vogt J, Amaral DG, Barnes CA (2007) EEG sharp waves and sparse ensemble unit activity in the macaque hippocampus. J Neurophysiol 98:898-910.

Strang G (2006) Linear algebra and its applications. Independence, $\mathrm{KY}$ : Cengage Learning.

Strichartz RS (2003) A guide to distribution theory and Fourier transforms. Singapore: World Scientific Publishing Company.

Strogatz SH (1994) Norbert Wiener's brain waves. In: Frontiers in mathematical biology, pp 122-138. New York: Springer.

Stumpf C (1965) Drug action on the electrical activity of the hippocampus. Int Rev Neurobiol 8:77-138.

Sullivan D, Csicsvari J, Mizuseki K, Montgomery S, Diba K, Buzsáki G (2011) Relationships between hippocampal sharp waves, ripples, and fast gamma oscillation: influence of dentate and entorhinal cortical activity. J Neurosci 31:8605-8616.

Tallon-Baudry C, Bertrand O, Delpuech C, Permier J (1997) Oscillatory gamma-band $(30-70 \mathrm{~Hz})$ activity induced by a visual search task in humans. J Neurosci 17:722-734.

Tamura M, Spellman TJ, Rosen AM, Gogos JA, Gordon JA (2017) Hippocampal-prefrontal theta-gamma coupling during performance of a spatial working memory task. Nat Commun 8:2182.

Terrazas A, Krause M, Lipa P, Gothard KM, Barnes CA, McNaughton BL (2005) Self-motion and the hippocampal spatial metric. J Neurosci 25:8085-8096. 
Tiitinen H, Sinkkonen J, Reinikainen K, Alho K, Lavikainen J, Näätänen R (1993) Selective attention enhances the auditory $40-\mathrm{Hz}$ transient response in humans. Nature 364:59-60.

Tort AB, Komorowski RW, Manns JR, Kopell NJ, Eichenbaum H (2009) Theta-gamma coupling increases during the learning of item-context associations. Proc Natl Acad Sci USA 106:2094220947.

Traub RD, Whittington MA, Colling SB, Buzsáki G, Jefferys JG (1996) Analysis of gamma rhythms in the rat hippocampus in vitro and in vivo. J Physiol 493:471-484.

Trimper JB, Stefanescu RA, Manns JR (2014) Recognition memory and theta-gamma interactions in the hippocampus. Hippocampus 24:341-353.

Vaidya SP, Johnston D (2013) Temporal synchrony and gamma-totheta power conversion in the dendrites of CA1 pyramidal neurons. Nat Neurosci 16:1812-1820.

Van Milligen BP, Sánchez E, Estrada T, Hidalgo C, Brañas B, Carreras B, García L (1995) Wavelet bicoherence: a new turbulence analysis tool. Phys Plasmas 2:3017-3032.

VIM (2004) International vocabulary of basic and general terms in metrology (VIM). Joint Commitee for Guides in Metorology, Ed 3. Geneva, Switzerland: International Organization.

Vladimirov VS (2002) Methods of the theory of generalized functions. Boca Raton, FL: CRC Press.

Wang J, Fang Y, Wang X, Yang H, Yu X, Wang H (2017) Enhanced gamma activity and cross-frequency interaction of resting-state electroencephalographic oscillations in patients with Alzheimer's disease. Front Aging Neurosci 9:243.

Watrous AJ, Tandon N, Conner CR, Pieters T, Ekstrom AD (2013) Frequency-specific network connectivity increases underlie accurate spatiotemporal memory retrieval. Nat Neurosci 16:349-356.

Welch P (1967) The use of fast Fourier transform for the estimation of power spectra: a method based on time averaging over short, modified periodograms. IEEE Trans Audio Electroacoust 15:7073.
Whishaw IQ, Vanderwolf CH (1973) Hippocampal EEG and behavior: changes in amplitude and frequency of RSA (theta rhythm) associated with spontaneous and learned movement patterns in rats and cats. Behav Biol 8:461-484.

Wiener N (1965) Cybernetics or control and communication in the animal and the machine. Cambridge, MA: The MIT Press.

Wiener N (1966) Nonlinear problems in random theory, pp 142. Cambridge, MA: The MIT Press.

Witter M, Amaral D (2004) Hippocampal formation. In: The rat nervous system (Paxinos G, ed). Houston, TX: Gulf Professional Publishing.

Wu Z, Huang NE (2009) Ensemble empirical mode decomposition: a noise-assisted data analysis method. Adv Adapt Data Anal 1:1-41.

Wulff $P$, Ponomarenko AA, Bartos $M$, Korotkova TM, Fuchs EC, Bähner F, Both M, Tort AB, Kopell NJ, Wisden W, Monyer H (2009) Hippocampal theta rhythm and its coupling with gamma oscillations require fast inhibition onto parvalbumin-positive interneurons. Proc Natl Acad Sci USA 106:3561-3566.

Ylinen A, Bragin A, Nádasdy Z, Jandó G, Szabó I, Sik A, Buzsáki G (1995) Sharp wave-associated high-frequency oscillation $(200 \mathrm{~Hz})$ in the intact hippocampus: network and intracellular mechanisms. J Neurosci 15:30-46.

Zhang X, Zhong W, Brankačk J, Weyer SW, Müller UC, Tort AB, Draguhn A (2016) Impaired theta-gamma coupling in APPdeficient mice. Sci Rep 6:21948.

Zheng C, Bieri KW, Trettel SG, Colgin LL (2015) The relationship between gamma frequency and running speed differs for slow and fast gamma rhythms in freely behaving rats. Hippocampus 25: 924-938.

Zheng C, Bieri KW, Hsiao YT, Colgin LL (2016) Spatial sequence coding differs during slow and fast gamma rhythms in the hippocampus. Neuron 89:398-408.

Zhong W, Ciatipis M, Wolfenstetter T, Jessberger J, Müller C, Ponsel S, Yanovsky Y, Brankačk J, Tort ABL, Draguhn A (2017) Selective entrainment of gamma subbands by different slow network oscillations. Proc Natl Acad Sci USA 114:4519-4524. 